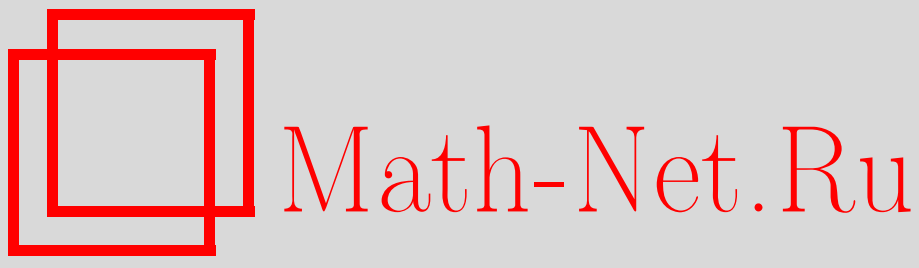

В. Ф. Бутузов, И. В. Неделько, О глобальной области влияния устойчивых решений с внутренними слоями в двумерном случае, Изв. РАН. Сер. матем., 2002, том 66, выпуск 1, 3-42

DOI: https://doi.org/10.4213/im369

Использование Общероссийского математического портала Math-Net.Ru подразумевает, что вы прочитали и согласны с пользовательским соглашением

http: //www. mathnet.ru/rus/agreement

Параметры загрузки:

IP : 54.162 .27 .143

26 апреля 2023 г., 13:56:33 


\title{
О глобальной области влияния устойчивых решений с внутренними слоями в двумерном случае
}

\begin{abstract}
Рассматривается начально-краевая задача для сингулярно возмущенного нестационарного уравнения реакции-диффузии с нелинейностью кубического типа. Наложенные условия обеспечивают существование устойчивого стационарного решения с внутренним переходным слоем в окрестности определенной кривой. Исследован вопрос о том, какие начальные функции принадлежат области влияния такого решения.

Библиограффия: 11 наименований.
\end{abstract}

\section{§ 1. Введение. Постановка задачи}

Рассмотрим задачу:

$$
\begin{aligned}
& \varepsilon^{2} \Delta u-u_{t}=f(u, x, \varepsilon), \quad(x, t) \in D \times(0,+\infty), \\
& \left.\frac{\partial u}{\partial n}\right|_{\partial D}=0, \quad t \in(0,+\infty), \\
& u(x, 0, \varepsilon)=u_{0}(x, \varepsilon), \quad x \in \bar{D},
\end{aligned}
$$

где $\varepsilon>0$ - мальй параметр, $x=\left(x_{1}, x_{2}\right), D \subset \mathbb{R}^{2}$ - ограниченная односвязная область с достаточно гладкой границей $\partial D, \frac{\partial}{\partial n}-$ производная по направлению внешней нормали к $\partial D$.

Предположим, что функция $f$ удовлетворяет следующему условию.

(A1) Существуют функции $\bar{u}(x)$ и $\hat{u}(x)$ из $C^{2}(\bar{D})$ такие, что $\bar{u}(x)<\hat{u}(x)$, $x \in \bar{D}$, и в области $\Pi=\{(u, x): \bar{u}(x) \leqslant u \leqslant \hat{u}(x), x \in \bar{D}\}$ функция $f(u, x, 0)$ обращается в нуль только на поверхностях $u=\varphi_{i}(x), i=0,1,2$, причем

$$
\begin{gathered}
\bar{u}(x)<\varphi_{1}(x)<\varphi_{0}(x) \equiv 0<\varphi_{2}(x)<\hat{u}(x), \quad x \in \bar{D}, \\
f_{u}\left(\varphi_{i}(x), x, 0\right)>0, \quad i=1,2 ; \quad f_{u}\left(\varphi_{0}(x), x, 0\right)<0, \quad x \in \bar{D} ;
\end{gathered}
$$

$f(u, x, \varepsilon)$ - достаточно гладкая функиия в области $\Pi_{1} \times\left[0, \varepsilon_{0}\right]$, әде $\Pi_{1}-$ некоторая открытая область, содержащая П, а $\varepsilon_{0}>0$ - некоторое число.

Нулевые условия в (1.2), а также предположение $\varphi_{0} \equiv 0$ взяты для простоты изложения. Все результаты несложно переформулировать для случая ненулевых краевых условий и $\varphi_{0} \not \equiv 0$.

Введем функцию

$$
J(x)=\int_{\varphi_{1}(x)}^{\varphi_{2}(x)} f(u, x, 0) d u .
$$

Работа выполнена при частичной финансовой поддержке РФФИ (проект № 99-01-01208).

(C) В.Ф. БУтУЗов, И. В. НедЕЛЬко, 2002 
(A2) Пусть существует достаточно зладкая замкнутая кривая $\Gamma \subset D$ без самопересечений, разделяющая область $D$ на две подобласти: $D_{0}^{(+)}($внутри кривой Г) и $D_{0}^{(-)}$, и такая, что

$$
J(x)=0, \quad \frac{\partial J}{\partial n_{0}}(x)<0, \quad x \in \Gamma,
$$

где $\frac{\partial}{\partial n_{0}}-$ производная по направлению нормали $к \Gamma$, направленной внутрь подобласти $D_{0}^{(+)}$.

Известно [1], [2], что при вьполнении условий (A1), (A2) для достаточно малых $\varepsilon$ существует стационарное решение $u_{s}(x, \varepsilon)$ задачи $(1.1),(1.2)$, имеющее внутренний переходный слой в окрестности кривой $\Gamma$, т.е. такое, что

$$
\lim _{\varepsilon \rightarrow 0} u_{s}(x, \varepsilon)= \begin{cases}\varphi_{1}(x), & x \in D_{0}^{(-)}, \\ \varphi_{2}(x), & x \in D_{0}^{(+)} .\end{cases}
$$

Решения такого типа назьвают контрастными структурами типа ступень$\kappa u$ (KCTC). Асимптотическое разложение КСТC можно построить методом пограничных функций [3], [4].

Анализ устойчивости двумерных КСТС проведен в работах [5], [6]. Из указанных работ следует, что при выполнении условий $(\mathrm{A} 1),(\mathrm{A} 2)$ решение $u_{s}(x, \varepsilon)$ задачи (1.1), (1.2) асимптотически устойчиво.

Одной из основных проблем как с теоретической, так и с прикладной точек зрения является ответ на вопрос о том, как устроено множество начальных функций $u_{0}(x, \varepsilon)($ см. $(1.3))$, из которых формируется при $t \rightarrow+\infty \operatorname{KCTC~} u_{s}(x, \varepsilon)$, если выполнены условия (A1), (A2) и $\varepsilon$ достаточно мало. Иными словами, как устроена область влияния стационарного решения $u_{s}(x, \varepsilon)$ в случае, когда оно асимптотически устойчиво.

В работе [6] показано, что области влияния $\mathrm{KCTC} u_{s}(x, \varepsilon)$ при условиях (A1), $(\mathrm{A} 2)$ и достаточно малых $\varepsilon$ принадлежат все функции $u_{0}(x, \varepsilon)$ (удовлетворяюшие определенным условиям гладкости и условию (1.2)) такие, что

$$
\left|u_{0}(x, \varepsilon)-u_{s}(x, \varepsilon)\right| \leqslant C \varepsilon^{2},
$$

где $C>0$ - некоторое фиксированное (при $\varepsilon \rightarrow 0$ ) число. Иными словами, было доказано, что область влияния решения $u_{s}(x, \varepsilon)$ содержит окрестность этого решения порядка $O\left(\varepsilon^{2}\right)$. Проблема усиления указанного результата, носящего локальный характер, в частности ответа на вопрос, может ли решение $u_{s}(x, \varepsilon)$ с фформироваться из начальной функции, отстоящей от него на величину порядка $O(1)$, оставалась открытой и была решена только для КСТС, возникаюших в одномерном аналоге $(D=[a, b])$ задачи $(1.1)-(1.3)[7]$.

Как и в соответствующем одномерном случае, рассмотренном в [7], в настоящей работе речь идет о глобальной области влияния $\mathrm{KCTC} u_{s}(x, \varepsilon)$, под которой будем понимать множество всех функций $u_{0}(x, \varepsilon)$, для каждой из которых $\exists \varepsilon^{\prime}>0$ такое, что при $\varepsilon \in\left(0, \varepsilon^{\prime}\right]$ решение задачи (1.1)-(1.3) сушествует и своим пределом при $t \rightarrow+\infty$ имеет $u_{s}(x, \varepsilon)$. Более точное определение глобальной области влияния дано в заключительном $\S 5$, где приведен основной результат данной работы - теорема 5.1 о глобальной области влияния $\mathrm{KCTC} u_{s}(x, \varepsilon)$. Чтобы сформулировать 
этот результат, введем еще одно требование. С этой целью выделим в области $D_{0}^{(+)}$все (отделенные друг от друга) множества и отдельные точки, если таковые имеются, где $J(x) \geqslant 0$, а в области $D_{0}^{(-)} \cup \partial D$ - все (отделенные друг от друга) множества и отдельные точки, если таковые имеются, где $J(x) \leqslant 0$. Класс (возможно, пустой) указанных элементов обозначим через $N$.

(А3) Пусть:

1) $J(x) \neq 0, \quad x \in \partial D$

2) если класс $N$ не пуст, то он содержит конечное число әлементов, причем любой әлемент класса $N$, не являющийся точкой, представляет из себя либо достаточно гладкую замкнутую кривую без самопересечений, либо область с достаточно гладкой гранищей.

Теорема 5.1 утверждает, что при выполнении условий (A1)-(А3) глобальной области влияния КСТС $u_{s}(x, \varepsilon)$ принадлежат все функции $u_{0}(x, \varepsilon)$, удовлетворяющие следующим двум условиям:

$$
\begin{gathered}
(\mathrm{A} 4) u_{0}(x, \varepsilon) \equiv u_{0}(x) \in C_{B}^{2}(\bar{D}) \equiv\left\{v(x): v(x) \in C^{2}(\bar{D}) ;\left.\frac{\partial v}{\partial n}\right|_{\partial D}=0\right\} u \\
\bar{u}(x)<u_{0}(x)<\hat{u}(x), \quad x \in \bar{D} .
\end{gathered}
$$

(A5) $\exists x^{(-)} \in D_{0}^{(-)} u \exists x^{(+)} \in D_{0}^{(+)}$maкue, ито:

$u_{0}\left(x^{(-)}\right)<0 \quad u \quad u_{0}(x)<0 \quad$ во всех точках $\quad x \in D_{0}^{(-)} \cup \partial D, \quad$ дде $\quad J(x) \leqslant 0 ;$

$u_{0}\left(x^{(+)}\right)>0 \quad u \quad u_{0}(x)>0 \quad$ во всех точках $\quad x \in D_{0}^{(+)}$, дде $J(x) \geqslant 0$.

Условие (А5), в частности, показывает, что начальная функция, из которой при $t \rightarrow+\infty$ формируется решение $u_{s}(x, \varepsilon)$, может иметь форму, весьма далекую от формы КСТС. Более того (см. следствие 5.1), если $J(x)>0$ при $x \in D_{0}^{(-)} \cup \partial D$ и $J(x)<0$ при $x \in D_{0}^{(+)}$, то в (1.6) и (1.7) можно оставить лишь требование сушествования хотя бы одной точки $x^{(-)} \in D_{0}^{(-)}$такой, что $u_{0}\left(x^{(-)}\right)<0$, и хотя бы одной точки $x^{(+)} \in D_{0}^{(+)}$такой, что $u_{0}\left(x^{(+)}\right)>0$.

Отметим, что если $\varphi_{0}(x) \not \equiv 0$, то неравенства в $(1.6)$ и $(1.7)$ для $u_{0}(x)$ нужно заменить соответственно на $u_{0}(x)<\varphi_{0}(x)$ и $u_{0}(x)>\varphi_{0}(x)$.

Чтобы оценить, насколько полно наш основной результат характеризует глобальную область влияния $\mathrm{KCTC} u_{s}(x, \varepsilon)$, в $\S 5$ приведена теорема 5.2 , показывающая, что установленные нами условия, обеспечивающие принадлежность начальной функции указанной области, близки к необходимым.

Скажем кратко о содержании других параграфов.

В $\S 2$ при условиях (A1), (А4) исследовано поведение решения задачи (1.1)-(1.3) на конечном промежутке времени, а также доказаны существование и ограниченность решения на бесконечном промежутке времени. 
В $\S 3$ получены более тонкие оценки на бесконечном промежутке времени. Сначала доказан ряд вспомогательных лемм, в которых представлены локальные оценки решения при выполнении требований (A1), (А4) и некоторых локальных условий на $J(x)$ и начальную функцию $u_{0}(x)$, а затем доказаны теорема 3.1 и усиливающая ее результат теорема 3.2 об оценках решения при выполнении всех требований (A1)-(A5).

В $\S 4$ с помошью теоремы 3.2 доказана теорема 4.1 о том, что пределом решения задачи (1.1)-(1.3) при $t \rightarrow \infty$ является $\mathrm{KCTC} u_{s}(x, \varepsilon)$.

Доказательства большинства вспомогательных и основных утверждений основано на построении барьеров для решения задачи (1.1)-(1.3).

В заключение отметим, что представленный в настоящей работе метод позволяет исследовать глобальные области влияния стационарных решений задачи (1.1), (1.2) без внутренних переходных слоев, т.е. решений $u^{(1)}(x, \varepsilon)$ и $u^{(2)}(x, \varepsilon)$, близких при малых $\varepsilon$ соответственно к $\varphi_{1}(x)$ и $\varphi_{2}(x)$ во всей области $\bar{D}$. Как известно, такие решения существуют в задаче $(1.1),(1.2)$ при выполнении условия (A1) для достаточно малых $\varepsilon$, причем являются асимптотически устойчивыми.

\section{§ 2. Лемма существования. Основные результаты для конечного промежутка времени}

2.1. Существование решения. Для точек $M\left(x_{1}, x_{2}\right)$ из малой окрестности $\partial D$ введем локальные координаты $(z, \ell)$, где $z$ - расстояние от точки $M$ до кривой $\partial D$ вдоль нормали к $\partial D$, которая проходит через $M$, а $\ell$ - координата той точки на $\partial D$, из которой выпушена указанная нормаль, $\ell \in\left[0, \ell_{0}\right], \ell_{0}$ - некоторое число. Известно (см., например, [3]), что сушествует число $\delta>0$ такое, что при $z \leqslant \delta$ соответствие между $(z, \ell)$ и $\left(x_{1}, x_{2}\right)$ взаимно однозначно.

ЛЕмма 2.1. Пусть выполнены условия (A1), (А4).

Тогда при достаточно мальх є задача (1.1)-(1.3) имеет единственное решение $u(x, t, \varepsilon) \in C^{1,0}(\bar{D} \times[0,+\infty)) \cap C^{2,1}(\bar{D} \times(0,+\infty))$, причем

$$
\bar{u}(x)-C \varepsilon<u(x, t, \varepsilon)<\hat{u}(x)+C \varepsilon, \quad(x, t) \in \bar{D} \times[0,+\infty),
$$

әде $C>0$ - некоторое число, не зависящее от $\varepsilon$.

У тверждение леммы 2.1 следует из [8], [9] и того факта, что функции $(\hat{u}+\varepsilon \widehat{P}(z, \varepsilon))$ и $(\bar{u}-\varepsilon \widehat{P}(z, \varepsilon))$, где

$$
\widehat{P}(z, \varepsilon)=\exp \left(-\frac{k z}{\varepsilon}\right) \zeta\left(\frac{z}{\delta}\right),
$$

$\zeta(y)$ - срезающая функция, $\zeta(y) \in C^{\infty}([0,+\infty)), \zeta(y)=1$ при $0 \leqslant y \leqslant 1 / 4$, $0 \leqslant \zeta(y) \leqslant 1$ при $1 / 4 \leqslant y \leqslant 1 / 2, \zeta(y)=0$ при $y \geqslant 1 / 2, \delta$ - указанное выше число, при достаточно большом $k>0$ и достаточно малых $\varepsilon$ являются соответственно верхним и нижним решениями задачи (1.1)-(1.3).

2.2. Асимптотическое приближение решения на конечном промежутке времени. Пусть выполнены условия (A1) и (А4). Асимптотику (нулевого порядка по $\varepsilon$ ) решения задачи (1.1)-(1.3) на промежутке $[0, T]$, где $T>0$ - произвольное, но фиксированное при $\varepsilon \rightarrow 0$ число, построим в виде

$$
U(x, t, \varepsilon)=\tilde{u}(x, t)+\varepsilon W(\xi, \ell, t) \zeta\left(\frac{z}{\delta}\right), \quad \xi=-\frac{z}{\varepsilon} .
$$


Функцию $\tilde{u}(x, t)$ определим как решение задачи ( $x$ играет роль параметра, $x \in \bar{D})$ :

$$
\begin{aligned}
& -\tilde{u}_{t}=f(\tilde{u}, x, 0), \quad t>0, \\
& \tilde{u}(x, 0)=u_{0}(x) .
\end{aligned}
$$

Из условий (A1) и (А4) следует, что решение $\tilde{u}(x, t)$ задачи $(2.3),(2.4)$ существует, единственно и

$$
\bar{u}(x)<\tilde{u}(x, t)<\hat{u}(x), \quad(x, t) \in \bar{D} \times[0,+\infty) .
$$

Определим теперь функцию

$$
W(\xi, \ell, t)=\frac{\partial \tilde{u}}{\partial z}(0, \ell, t) \exp (\xi)
$$

где $\tilde{u}(z, \ell, t) \equiv \tilde{u}(x(z, \ell), t)$.

По построению функция $U(x, t, \varepsilon)$ удовлетворяет следуюшим соотношениям:

$$
\begin{aligned}
& \varepsilon^{2} \Delta U-U_{t}= f(U, x, \varepsilon)+O(\varepsilon), \quad(x, t) \in D \times(0, T], \\
&\left.\frac{\partial U}{\partial n}\right|_{\partial D}=0, \quad t \in(0, T], \\
& U(x, 0, \varepsilon)=u_{0}(x), \quad x \in \bar{D} \\
& U(x, t, \varepsilon)=\tilde{u}(x, t)+O(\varepsilon), \quad(x, t) \in \bar{D} \times[0, T] .
\end{aligned}
$$

ЛЕмма 2.2. Пусть выполнены условия (A1) и (A4). Тогда для любого $T>0$, фиксированного при $\varepsilon \rightarrow 0$, существует число $C>0$ такое, что при достаточно мальх в решение $u(x, t, \varepsilon)$ задачи (1.1)-(1.3) удовлетворяет неравенству

$$
|u(x, t, \varepsilon)-\tilde{u}(x, t)| \leqslant C \varepsilon, \quad(x, t) \in \bar{D} \times[0, T] .
$$

ДокАЗАТЕЛЬСтво. Пусть $T>0$ фиксировано. Рассмотрим функцию

$$
w(x, t, \varepsilon)=(u(x, t, \varepsilon)-U(x, t, \varepsilon)) \exp (-A t),
$$

где число $A>0$ выбрано так, что

$$
B(x, t, \varepsilon)=\int_{0}^{1} f_{u}(U(x, t, \varepsilon)+(u(x, t, \varepsilon)-U(x, t, \varepsilon)) s, x, \varepsilon) d s+A>1
$$

при $(x, t) \in \bar{D} \times[0, T]$ и малых $\varepsilon$. В силу леммы $2.1,(2.5)$ и (2.9) такой выбор $A$ возможен. Из (1.1)-(1.3) и (2.6)-(2.8) получаем

$$
\begin{gathered}
\varepsilon^{2} \Delta w-B(x, t, \varepsilon) w-w_{t}=O(\varepsilon), \quad(x, t) \in D \times(0, T] \\
\left.\frac{\partial w}{\partial n}\right|_{\partial D}=0, \quad t \in(0, T] \\
w(x, 0, \varepsilon)=0, \quad x \in \bar{D} .
\end{gathered}
$$

Отсюда следует, что

$$
|w(x, t, \varepsilon)| \leqslant C_{0} \varepsilon, \quad(x, t) \in \bar{D} \times[0, T],
$$

где $C_{0}>0$ - некоторое число, а потому в силу $(2.9)$ существует $C>0$ такое, что при достаточно малых $\varepsilon$ выполнено (2.10). Лемма доказана. 


\section{3. Основные результаты.}

ЛЕмма 2.3. Пусть выполнены условия (A1), (A4), и пусть существует область $\Omega^{(-)} \subseteq D\left(\Omega^{(+)} \subseteq D\right)$ такая, ито

$$
u_{0}(x)<0, \quad x \in \bar{\Omega}^{(-)} \quad\left(u_{0}(x)>0, \quad x \in \bar{\Omega}^{(+)}\right) .
$$

Тогда для любого $\eta>0$ существует $T_{*}=T_{*}(\eta)>0$ такое, что для любого $T \geqslant T_{*}$ при достаточно мальх в решение $u(x, t, \varepsilon)$ задачи (1.1)-(1.3) удовлетворяет неравенствам

$$
\begin{gathered}
u(x, t, \varepsilon)<\varphi_{1}(x)+\eta, \quad(x, t) \in \bar{\Omega}^{(-)} \times\left[T_{*}, T\right] \\
\left(u(x, t, \varepsilon)>\varphi_{2}(x)-\eta, \quad(x, t) \in \bar{\Omega}^{(+)} \times\left[T_{*}, T\right]\right), \\
\varphi_{1}(x)-\eta<u(x, t, \varepsilon)<\varphi_{2}(x)+\eta, \quad(x, t) \in \bar{D} \times\left[T_{*}, T\right] .
\end{gathered}
$$

ДоКАЗАТЕЛЬСТво. В силу леммы 2.2 достаточно доказать, что для любого $\eta>0$ сушествует $T_{*}>0$ такое, что неравенства $(2.11),(2.12)$ выполняются при $t \geqslant T_{*}$ для функции $\tilde{u}(x, t)$ (т.е. при $t \geqslant T_{*}$ имеют место неравенства $(2.11),(2.12)$, в которых $u$ заменено на $\tilde{u}$ ). Докажем, например, что по заданному $\eta>0$ можно указать $T_{*}>0$ такое, что

$$
\tilde{u}(x, t)<\varphi_{1}(x)+\eta, \quad(x, t) \in \bar{\Omega}^{(-)} \times\left[T_{*},+\infty\right) .
$$

Остальные неравенства (2.11), (2.12) для $\tilde{u}$ доказываются аналогично.

Зафикисируем произвольное $\eta>0$. Пусть

$$
h=\min \left\{\eta ;-\frac{1}{2} \max _{\bar{\Omega}^{(-)}} u_{0}(x) ;-\frac{1}{2} \max _{\bar{\Omega}^{(-)}} \varphi_{1}(x)\right\} .
$$

Введем функции

$$
\begin{aligned}
v_{1}(x, t) & =-h+\frac{t}{T_{*}}\left(\varphi_{1}(x)+\frac{3}{2} h\right), & (x, t) & \in \bar{\Omega}^{(-)} \times\left[0, T_{*}\right], \\
v_{2}(x) & =\varphi_{1}(x)+\frac{1}{2} h, & (x, t) & \in \bar{\Omega}^{(-)} \times\left[T_{*},+\infty\right),
\end{aligned}
$$

где

$$
\begin{gathered}
T_{*}=\frac{2 \max _{\bar{D}}\left|\varphi_{1}(x)\right|+3 h}{m}, \quad m=\min _{\Pi^{(+)}} f(u, x, 0)>0, \\
\Pi^{(+)}=\left\{(u, x): \varphi_{1}(x)+\frac{1}{2} h \leqslant u \leqslant-h, \quad x \in \bar{\Omega}^{(-)}\right\} .
\end{gathered}
$$

При таком выборе $T_{*}$, как показывают простые вычисления,

$$
\frac{\partial v_{1}}{\partial t}+f\left(v_{1}, x, 0\right) \geqslant \frac{1}{2} m, \quad(x, t) \in \bar{\Omega}^{(-)} \times\left[0, T_{*}\right] .
$$


Используя это неравенство и уравнение (2.3), получаем

$$
\frac{\partial\left(v_{1}-\tilde{u}\right)}{\partial t}+f\left(v_{1}, x, 0\right)-f(\tilde{u}, x, 0) \geqslant \frac{1}{2} m>0
$$

или

$$
\frac{\partial\left(v_{1}-\tilde{u}\right)}{\partial t}+f_{u}^{*} \cdot\left(v_{1}-\tilde{u}\right)>0, \quad(x, t) \in \bar{\Omega}^{(-)} \times\left[0, T_{*}\right] .
$$

Отсюда, учитывая начальное условие

$$
\left.\left(v_{1}-\tilde{u}\right)\right|_{t=0}=-h-u_{0}(x)>0, \quad x \in \bar{\Omega}^{(-)},
$$

приходим к неравенству

$$
v_{1}-\tilde{u}>0, \quad(x, t) \in \bar{\Omega}^{(-)} \times\left[0, T_{*}\right],
$$

и, следовательно,

т.е.

$$
\left.\tilde{u}\right|_{t=T_{*}}<\left.v_{1}\right|_{t=T_{*}}=\varphi_{1}(x)+\frac{1}{2} h=v_{2}(x), \quad x \in \bar{\Omega}^{(-)},
$$

$$
\left.\left(v_{2}-\tilde{u}\right)\right|_{t=T_{*}}>0, \quad x \in \bar{\Omega}^{(-)} .
$$

Для $v_{2}-\tilde{u}$ аналогично (2.14) получаем

$$
\frac{\partial\left(v_{2}-\tilde{u}\right)}{\partial t}+f_{u}^{* *} \cdot\left(v_{2}-\tilde{u}\right) \geqslant \frac{1}{2} m>0, \quad(x, t) \in \bar{\Omega}^{(-)} \times\left[T_{*},+\infty\right) .
$$

Отсюда и из (2.15) следует, что

$$
v_{2}-\tilde{u}>0, \quad(x, t) \in \bar{\Omega}^{(-)} \times\left[T_{*},+\infty\right),
$$

и, значит,

$$
\tilde{u}(x, t)<v_{2}(x)=\varphi_{1}(x)+h \leqslant \varphi_{1}(x)+\eta, \quad(x, t) \in \bar{\Omega}^{(-)} \times\left[T_{*},+\infty\right) .
$$

Тем самым неравенство (2.13) и лемма доказаны.

СлЕДСТВИЕ 2.1. Лемма 2.3 останется в силе, если в (2.12) заменить $\left[T_{*}, T\right] н а\left[T_{*},+\infty\right)$.

Действительно, для любого $\eta>0$ возьмем $T_{*}=T_{*}(\eta / 2)>0$. Тогда при достаточно малых $\varepsilon$ в силу (2.12) будут выполнены неравенства

$$
\varphi_{1}(x)-\frac{\eta}{2}<u\left(x, T_{*}, \varepsilon\right)<\varphi_{2}(x)+\frac{\eta}{2}, \quad x \in \bar{D} .
$$

Положим

$$
\hat{v}(x, \varepsilon)=\varphi_{2}(x)+\frac{\eta}{2}+\varepsilon \widehat{P}(z, \varepsilon), \quad \bar{v}(x, \varepsilon)=\varphi_{1}(x)-\frac{\eta}{2}-\varepsilon \widehat{P}(z, \varepsilon),
$$

где функция $\widehat{P}(z, \varepsilon)$ определена формулой $(2.1)$. Для любого достаточно малого $\eta$ при достаточно малых $\varepsilon$ и достаточно большом $k$ (см. $(2.1))$ функции $\hat{v}(x, \varepsilon)$ и $\bar{v}(x, \varepsilon)$ являются верхним и нижним барьерами для решения $u(x, t, \varepsilon)$ при $t \geqslant T_{*}$, т.e.

$$
\bar{v}(x, \varepsilon)<u(x, t, \varepsilon)<\hat{v}(x, \varepsilon), \quad(x, t) \in \bar{D} \times\left[T_{*},+\infty\right) .
$$

Отсюда, взяв $\varepsilon$ столь малым, что $\varepsilon \widehat{P}(z, \varepsilon)<\eta / 2$, приходим к неравенствам $(2.12)$ для $(x, t) \in \bar{D} \times\left[T_{*},+\infty\right)$.

Из леммы 2.3 и следствия 2.1 непосредственно вытекает следуюший результат. 
ТЕОРема 2.1. Пусть выполнены условия (A1), (A4), и пусть существует область $\Omega^{(-)} \subseteq D\left(\Omega^{(+)} \subseteq D\right)$ такая, что

$$
u_{0}(x)<0, \quad x \in \bar{\Omega}^{(-)} \quad\left(u_{0}(x)>0, \quad x \in \bar{\Omega}^{(+)}\right) .
$$

Тогда для любого $\eta>0$ существует $T_{0}=T_{0}(\eta)>0$ maкое, что для любого $T \geqslant T_{0}$ при достаточно мальх в решение $u(x, t, \varepsilon)$ задачи (1.1)-(1.3) удовлетворяет неравенствам

$$
\begin{aligned}
u(x, t, \varepsilon) & <\varphi_{1}(x)+\eta, & & (x, t) \in \bar{\Omega}^{(-)} \times\left[T_{0}, T\right] \\
(u(x, t, \varepsilon) & >\varphi_{2}(x)-\eta, & & \left.(x, t) \in \bar{\Omega}^{(+)} \times\left[T_{0}, T\right]\right), \\
\varphi_{1}(x)-\eta<u(x, t, \varepsilon) & <\varphi_{2}(x)+\eta, & & (x, t) \in \bar{D} \times\left[T_{0},+\infty\right) .
\end{aligned}
$$

\section{§ 3. Поведение решения на бесконечном промежутке времени}

\section{1. Вспомогательные леммы.}

ЛЕмма 3.1. Пусть выполнены условия (A1), (А4) и существует открытый круг $K$ с иентром в некоторой точке $x^{(-)}\left(x^{(+)}\right)$такой, что $\bar{K} \subset D$ $u$

$$
\begin{array}{cl}
J(x)>0, \quad x \in \bar{K} & (J(x)<0, \quad x \in \bar{K}), \\
u_{0}\left(x^{(-)}\right)<0 & \left(u_{0}\left(x^{(+)}\right)>0\right) .
\end{array}
$$

Тогда для любого $\eta>0$ при достаточно мальх в существует $T=$ $T(\eta, \varepsilon)>0$ такое, что решение $u(x, t, \varepsilon)$ задачи (1.1)-(1.3) удовлетворяет неравенству

$$
\begin{aligned}
& u(x, t, \varepsilon)<\varphi_{1}(x)+\eta, \quad(x, t) \in \bar{K} \times[T,+\infty) \\
& \left(u(x, t, \varepsilon)>\varphi_{2}(x)-\eta, \quad(x, t) \in \bar{K} \times[T,+\infty)\right) .
\end{aligned}
$$

ДокАЗАтЕльство. Докажем утверждение леммы, относяшееся к неравенству (3.1). Утверждение, относящееся к неравенству (3.2), доказывается аналогично. Далее буквой $R$ обозначаем радиус круга $K$.

Пусть $K_{0}-$ круг некоторого радиуса $a$ с центром в точке $x^{(-)}$такой, что

$$
\bar{K}_{0} \subset K, \quad u_{0}(x)<0, \quad x \in \bar{K}_{0} .
$$

Существование $K_{0}$ следует из неравенства $u_{0}\left(x^{(-)}\right)<0$ и непрерывности $u_{0}(x)$.

Введем полярную систему координат $(\rho, \sigma)$ с центром в точке $x^{(-)}$. Пусть число $b \in(0, a)$ таково, что для круга $K_{1}$ радиуса $R+b$ с центром в точке $x^{(-)}$выполнены соотношения

$$
\bar{K}_{1} \subset D, \quad J(x)>0, \quad x \in \bar{K}_{1} .
$$

Введем семейство окружностей $\Gamma_{\gamma}, \gamma \in[0,1]$, задав его в координатах $(\rho, \sigma)$ в виде

$$
\rho=a+\gamma(R+b-a) \equiv R_{\gamma}
$$


Очевидно, что

$$
\Gamma_{\gamma} \in \overline{K_{1} \backslash K_{0}}, \quad \gamma \in[0,1],
$$

окружность $\Gamma_{0}$ является границей круга $K_{0}$, а окружность $\Gamma_{1}-$ границей круга $K_{1}$.

Через $K_{\gamma}$ обозначим круг, ограниченный $\Gamma_{\gamma}$.

Введем обозначения: $f(u, \rho, \sigma, \varepsilon) \equiv f(u, x(\rho, \sigma), \varepsilon), \varphi_{i}(\rho, \sigma) \equiv \varphi_{i}(x(\rho, \sigma))$.

Определим функцию

$$
\beta(x, \gamma, \mu)=\varphi_{1}(x)+\mu+Q_{0}\left(\tau_{\gamma}, \sigma, \gamma\right)+\mu Q_{1}\left(\tau_{\gamma}, \sigma, \gamma\right), \quad x \in \bar{K}_{\gamma}, \quad \gamma \in[0,1],
$$

где $\mu>0$ - число, которое уточним ниже, $\tau_{\gamma}=\left(R_{\gamma}-\rho\right) / \varepsilon$.

Функция $Q_{0}\left(\tau_{\gamma}, \sigma, \gamma\right)$ является решением задачи ( $\gamma$ играет роль параметра, $\gamma \in$ $[0,1])$ :

$$
\begin{gathered}
\frac{\partial^{2}}{\partial \tau_{\gamma}^{2}} Q_{0}=f\left(\varphi_{1}\left(R_{\gamma}, \sigma\right)+Q_{0}, R_{\gamma}, \sigma, 0\right), \quad \tau_{\gamma}>0, \\
Q_{0}(0, \sigma, \gamma)=\varphi_{2}\left(R_{\gamma}, \sigma\right)-\varphi_{1}\left(R_{\gamma}, \sigma\right), \quad Q_{0}(+\infty, \sigma, \gamma)=0 .
\end{gathered}
$$

Так как в силу $(3.3),(3.4) J(x)>0$ при $x \in \Gamma_{\gamma}, \gamma \in[0,1]$, то $Q_{0}$ определяется однозначно, является положительной монотонно убывающей функцией $\tau_{\gamma}$ при $\tau_{\gamma} \geqslant 0$ и имеет экспоненциальные оценки по переменной $\tau_{\gamma}$ сверху и снизу, причем эти оценки равномерны по $\gamma \in[0,1]$ и $\sigma \in[0,2 \pi]$, т.е. существуют постоянные $C_{i}>0$ и $m_{i}>0, i=1,2$, не зависяшие от $\tau_{\gamma}, \gamma$ и $\sigma$ и такие, что

$$
\begin{array}{ll}
\left|Q_{0}\left(\tau_{\gamma}, \sigma, \gamma\right)\right| \leqslant C_{1} \exp \left(-m_{1} \tau_{\gamma}\right), \quad \tau_{\gamma} \geqslant 0, \\
\left|Q_{0}\left(\tau_{\gamma}, \sigma, \gamma\right)\right| \geqslant C_{2} \exp \left(-m_{2} \tau_{\gamma}\right), \quad \tau_{\gamma} \geqslant 0 .
\end{array}
$$

Функцию $Q_{1}\left(\tau_{\gamma}, \sigma, \gamma\right)$ определим как решение задачи

$$
\begin{aligned}
& \frac{\partial^{2}}{\partial \tau_{\gamma}^{2}} Q_{1}=f_{u}\left(\tau_{\gamma}, \sigma, \gamma\right) Q_{1}+f_{1}\left(\tau_{\gamma}, \sigma, \gamma\right), \quad \tau_{\gamma}>0, \\
& Q_{1}(0, \sigma, \gamma)=0, \quad Q_{1}(+\infty, \sigma, \gamma)=0,
\end{aligned}
$$

где $f_{u}\left(\tau_{\gamma}, \sigma, \gamma\right)=f_{u}\left(\varphi_{1}\left(R_{\gamma}, \sigma\right)+Q_{0}, R_{\gamma}, \sigma, 0\right), f_{1}\left(\tau_{\gamma}, \sigma, \gamma\right)=\left[f_{u}\left(\tau_{\gamma}, \sigma, \gamma\right)-\right.$ $\left.f_{u}\left(\varphi_{1}\left(R_{\gamma}, \sigma\right), R_{\gamma}, \sigma, 0\right)\right]$.

Функция $Q_{1}$ допускает явное представление [4] и имеет равномерную по $\gamma \in$ $[0,1]$ и $\sigma \in[0,2 \pi]$ экспоненциальную оценку типа $(3.5)$.

Домножим функции $Q_{i}, i=1,2$, на срезаюшую функцию $\zeta\left(\left(R_{\gamma}-\rho\right) / b\right.$ ) (функция $\zeta$ была определена в $\S 2)$ и получившиеся в результате этой процедуры функции снова обозначим через $Q_{i}, i=1,2$.

По самому построению функция $\beta(x, \gamma, \mu)$ удовлетворяет соотношению

$$
\varepsilon^{2} \Delta \beta-f(\beta, x, \varepsilon)=-\mu f_{u}\left(\varphi_{1}(x), x, 0\right)+O(\varepsilon)+O\left(\mu^{2}\right), \quad x \in \bar{K}_{\gamma}, \quad \gamma \in[0,1],
$$

откуда следует, что существует постоянная $C_{3}>0$, не зависящая от $\varepsilon, \mu, \gamma$ и такая, что для любого достаточно малого $\mu$ при достаточно малых $\varepsilon$ выполняется неравенство

$$
\varepsilon^{2} \Delta \beta-f(\beta, x, \varepsilon)<-C_{3} \mu, \quad x \in \bar{K}_{\gamma}, \quad \gamma \in[0,1]
$$


Используя равномерные экспоненциальные оценки функций $Q_{i}$, нетрудно доказать, что для любого достаточно малого $\mu$ при достаточно малых $\varepsilon$ имеет место неравенство

$$
\beta(x, \gamma, \mu)>\varphi_{1}(x)+\frac{\mu}{2}, \quad x \in \bar{K}_{\gamma}, \quad \gamma \in[0,1] .
$$

Отметим также очевидное равенство

$$
\beta(x, \gamma, \mu)=\varphi_{2}(x)+\mu, \quad x \in \Gamma_{\gamma}, \quad \gamma \in[0,1],
$$

и воспользуемся теоремой 2.1 , согласно которой сушествует $T_{0}=T_{0}(\mu / 2)>0$ такое, что при достаточно малых $\varepsilon$ решение $u(x, t, \varepsilon)$ задачи (1.1)-(1.3) удовлетворяет неравенствам

$$
\begin{aligned}
u\left(x, T_{0}, \varepsilon\right) & <\varphi_{1}(x)+\frac{\mu}{2}, \quad x \in \bar{K}_{0}, \\
u(x, t, \varepsilon) & <\varphi_{2}(x)+\mu, \quad(x, t) \in \bar{D} \times\left[T_{0},+\infty\right) .
\end{aligned}
$$

Введем функцию

$w(x, t, \mu, \varepsilon)=[\beta(x, \gamma(t), \mu)-u(x, t, \varepsilon)] \exp \left(-A\left(t-T_{0}\right)\right), \quad x \in \bar{K}_{\gamma(t)}, \quad t \in\left[T_{0}, T\right]$,

где

$$
\gamma(t)=\frac{t-T_{0}}{T-T_{0}}
$$

а величины $A$ и $T=T(\mu, \varepsilon)$ уточним ниже. Очевидно, что $0 \leqslant \gamma(t) \leqslant 1$ при $t \in\left[T_{0}, T\right], \gamma\left(T_{0}\right)=0, \gamma(T)=1$. Используя $(3.7)$, нетрудно получить неравенство $\varepsilon^{2} \Delta w-w_{t}-B w<\left(-C_{3} \mu-\frac{\partial \beta}{\partial \gamma} \cdot \frac{1}{T-T_{0}}\right) \exp \left(-A\left(t-T_{0}\right)\right), \quad x \in \bar{K}_{\gamma(t)}, \quad t \in\left[T_{0}, T\right]$,

где $B=B(x, t, \varepsilon, \mu)=A+\int_{0}^{1} f_{u}(u+(\beta-u) s, x, \varepsilon) d s$, и, следовательно, $B>0$, если взять достаточно большое $A>0$.

Из вида $\beta(x, \gamma, \mu)$ следует, что $\frac{\partial \beta}{\partial \gamma}$ имеет оценку

$$
\left|\frac{\partial \beta}{\partial \gamma}\right| \leqslant \frac{C}{\varepsilon}
$$

где постоянная $C>0$ не зависит от $\varepsilon, \gamma, \mu$ (для ограниченного интервала изменения $\mu$ ). Поэтому если взять $T=T(\mu, \varepsilon)$ такое, что

$$
T>T_{0}+\frac{C}{C_{3} \mu \varepsilon},
$$

то для любого достаточно малого $\mu$ при достаточно малых $\varepsilon$ будет выполнено неравенство

$$
\varepsilon^{2} \Delta w-w_{t}-B w<0, \quad x \in \bar{K}_{\gamma(t)}, \quad t \in\left[T_{0}, T\right] .
$$

Из (3.8) и (3.10) имеем

$$
w\left(x, T_{0}, \mu, \varepsilon\right)>0, \quad x \in \bar{K}_{\gamma\left(T_{0}\right)},
$$


а из (3.9) и (3.11) получаем

$$
w(x, t, \mu, \varepsilon)>0, \quad x \in \Gamma_{\gamma(t)}, \quad t \in\left[T_{0}, T\right] .
$$

Из (3.12)-(3.14) очевидным образом следует, что $w(x, t, \mu, \varepsilon)>0, x \in \bar{K}_{\gamma(t)}$, $t \in\left[T_{0}, T\right]$, в частности, при $t=T$ имеем неравенство

$$
w(x, T, \mu, \varepsilon)>0, \quad x \in \bar{K}_{\gamma(T)}=\bar{K}_{1},
$$

откуда получаем

$$
u(x, T, \varepsilon)<\beta(x, \gamma(T), \mu)=\beta(x, 1, \mu), \quad x \in \bar{K}_{1} .
$$

Из (3.9) и (3.11) следует, что

$$
u(x, t, \varepsilon)<\beta(x, 1, \mu), \quad x \in \Gamma_{1}, \quad t \in[T,+\infty) .
$$

Неравенства (3.15), (3.16) и неравенство (3.7), взятое при $\gamma=1$, показывают, что функция $\beta(x, 1, \mu)$ является стационарным верхним барьером для решения $u(x, t, \varepsilon)$ при $(x, t) \in \bar{K}_{1} \times[T,+\infty)$, т.е.

$$
u(x, t, \varepsilon)<\beta(x, 1, \mu), \quad(x, t) \in \bar{K}_{1} \times[T,+\infty) .
$$

Рассматривая выражение для $\beta(x, 1, \mu)$ и учитывая, что функции $Q_{i}, i=1,2$, домножены на срезающую функцию, приходим к выводу, что выполнено равенство

$$
\beta(x, 1, \mu)=\varphi_{1}(x)+\mu, \quad x \in \bar{K} .
$$

Возьмем теперь столь малое $\eta>0$, чтобы для $\mu=\eta$ при достаточно малых $\varepsilon$ было выполнено неравенство (3.17). Тогда из (3.17) и (3.18) получим неравенство (3.1):

$$
u(x, t, \varepsilon)<\varphi_{1}(x)+\eta, \quad(x, t) \in \bar{K} \times[T,+\infty) .
$$

Лемма доказана.

Следуюшая лемма обобщает результат леммы 3.1 на случай, когда берется не круг $K$, а произвольная область $D^{(-)}\left(D^{(+)}\right)$, замыкание которой лежит внутри $D$.

ЛЕмма 3.2. Пусть выполнены условия (A1), (A4) и $D^{(-)}\left(D^{(+)}\right)$- область maкая, umo $\bar{D}^{(-)} \subset D\left(\bar{D}^{(+)} \subset D\right) u$

$$
J(x)>0, \quad x \in \bar{D}^{(-)} \quad\left(J(x)<0, \quad x \in \bar{D}^{(+)}\right) .
$$

Пусть, далее, существует точка $\bar{x}^{(-)} \in D^{(-)}\left(\bar{x}^{(+)} \in D^{(+)}\right)$такая, что $u_{0}\left(\bar{x}^{(-)}\right)<0 \quad\left(u_{0}\left(\bar{x}^{(+)}\right)>0\right)$.

Тогда для любого $\eta>0$ при достаточно мальх $\varepsilon$ существует $T_{1}=$ $T_{1}(\eta, \varepsilon)>0$ такое, что решение $u(x, t, \varepsilon)$ задачи (1.1)-(1.3) удовлетворяет неравенствам

$$
\begin{aligned}
u(x, t, \varepsilon)<\varphi_{1}(x)+\eta, & & (x, t) \in \bar{D}^{(-)} \times\left[T_{1},+\infty\right) \\
\left(u(x, t, \varepsilon)>\varphi_{2}(x)-\eta,\right. & & \left.(x, t) \in \bar{D}^{(+)} \times\left[T_{1},+\infty\right)\right) .
\end{aligned}
$$


ДокаЗАТЕльство. Докажем утверждение леммы, относящееся к неравенству (3.19). Утверждение, относящееся к неравенству (3.20), доказывается аналогично.

Пусть число $h>0$ столь мало, что для области $D^{h}$, представляющей собой объединение $D^{(-)}$с $h$-окрестностью ее гранищы $\partial D^{(-)}$, выполнены соотношения

$$
\bar{D}^{h} \subset D, \quad J(x)>0, \quad x \in \bar{D}^{h} .
$$

Пусть $\left\{E_{i}^{h / 4}\right\}, i=1,2, \ldots, n$ (где $n$ - некоторое натуральное число) - множество открытых кругов радиуса $h / 4$ с центрами в некоторых точках $x_{i} \in D$ такое, что

$$
D^{(-)} \subset \bigcup_{i=1}^{n} E_{i}^{h / 4}
$$

и для любого $j \in \overline{1, n}$ имеем $\bar{E}_{j}^{h / 4} \cap \bar{D}^{(-)} \neq \varnothing$. Тогда

$$
\bigcup_{i=1}^{n} \bar{E}_{i}^{h / 4} \subset D^{h}
$$

Покажем, что $\forall \eta>0$ и $\forall j \in \overline{1, n}$ при достаточно малых $\varepsilon$ найдется $T_{(j)}>0$ такое, что решение $u(x, t, \varepsilon)$ задачи (1.1)-(1.3) удовлетворяет неравенству

$$
u(x, t, \varepsilon)<\varphi_{1}(x)+\eta, \quad(x, t) \in \bar{E}_{j}^{h / 4} \times\left[T_{(j)},+\infty\right) .
$$

Зафиксируем произвольное $\eta>0$ и $j \in \overline{1, n}$. Если $x_{j}=\bar{x}^{(-)}$, то (3.23) следует из леммы 3.1.

Пусть $x_{j} \neq \bar{x}^{(-)}$. Соединим точку $x_{j}$ с точкой $\bar{x}^{(-)}$непрерьвной спрямляемой кривой $S$, лежащей вместе со своей $d$-окрестностью (где $d>0$-некоторое число) внутри $D^{h}$. Пусть $s$-координата точки $M$ на кривой $S$, равная расстоянию между $\bar{x}^{(-)}$и $M$ вдоль $S$. Выберем целое число $m \geqslant 0$ так, чтобы разность между длиной кривой $S$ и числом $s_{m}=(m / 2) d$ была неотрицательной и не превосходила $d / 2$. Пусть $V_{k}, k=0,1, \ldots, m,-$ круги радиуса $d$ с центрами в точках $M_{k}$ кривой $S$ с координатами $s_{k}=(k d) / 2$. Без ограничения обшности можно считать, что $M_{m} \neq$ $x_{j}$ (случай $M_{m}=x_{j}$ рассматривается аналогично). Через $V_{m+1}$ обозначим круг $E_{j}^{h / 4}$. Отметим, что центром $V_{0}$ является $\bar{x}^{(-)}, \forall k \in \overline{1, m+1}$ центр круга $V_{k}$ лежит в круге $V_{k-1}$ и $\bar{V}_{k} \subset \bar{D}^{h}, k=0,1, \ldots, m+1$, а значит, вследствие $(3.21)$

$$
J(x)>0, \quad x \in \bar{V}_{k} \subset D, \quad k=0,1, \ldots, m+1 .
$$

Так как $u_{0}\left(\bar{x}^{(-)}\right)<0$, то в силу $(3.24)$ для круга $V_{0}$ выполнены условия леммы 3.1. Используя лемму 3.1 и теорему 2.1 , получаем, что для любого $\mu>0$ при достаточно малых $\varepsilon$ существует $t_{0}=t_{0}(\mu, \varepsilon)>0$ такое, что решение $u(x, t, \varepsilon)$ задачи (1.1)-(1.3) удовлетворяет неравенствам

$$
\begin{array}{ll}
u(x, t, \varepsilon)<\varphi_{1}(x)+\mu / 2, & (x, t) \in \bar{V}_{0} \times\left[t_{0},+\infty\right), \\
u(x, t, \varepsilon)<\varphi_{2}(x)+\mu, & (x, t) \in \bar{D} \times\left[t_{0},+\infty\right) .
\end{array}
$$


Введем обозначения: $x^{(-)}=M_{1}, K=V_{1}$. Так как центр $x^{(-)}$круга $K$ лежит внутри $V_{0}$ и выполнено неравенство (3.25), можно утверждать, что найдется круг $K_{0}$ с центром в $x^{(-)}$такой, что $\bar{K}_{0} \subset K$,

$$
u\left(x, t_{0}, \varepsilon\right)<\varphi_{1}(x)+\mu / 2, \quad x \in \bar{K}_{0} .
$$

Обозначим через $R$ радиус круга $K$, через $a$-радиус круга $K_{0}$. Пользуясь тем, что в силу (3.24) $J(x)>0, x \in \bar{K} \subset D$, введем полярные координаты $(\rho, \sigma)$ с центром в точке $x^{(-)}$, число $b$, семейство $\Gamma_{\gamma}$ и функцию $\beta(x, \gamma, \mu)$ точно так же, как в доказательстве леммы 3.1. Теперь с помошью функции $\beta(x, \gamma, \mu)$, используя неравенства (3.27) и (3.26) вместо $(3.10)$ и (3.11) (с заменой $T_{0}$ на $\left.t_{0}\right)$, тем же путем, что и при доказательстве леммы 3.1 , приходим к выводу, что для любого достаточно малого $\eta>0$ при достаточно малых $\varepsilon$ существует $T=T(\eta, \varepsilon)>0$ такое, что

$$
u(x, t, \varepsilon)<\varphi_{1}(x)+\eta, \quad(x, t) \in \bar{K} \times[T,+\infty) .
$$

Отсюда и из (3.26) следует, что $\forall \mu>0$ при достаточно малых $\varepsilon$ можно указать такое $t_{1}=t_{1}(\mu, \varepsilon)>0$, что справедливы неравенства

$$
\begin{array}{lll}
u(x, t, \varepsilon)<\varphi_{1}(x)+\mu / 2, & (x, t) \in \bar{V}_{1} \times\left[t_{1},+\infty\right), \\
u(x, t, \varepsilon)<\varphi_{2}(x)+\mu, & (x, t) \in \bar{D} \times\left[t_{1},+\infty\right) .
\end{array}
$$

Если $m>0$, то уже на основании (3.28), (3.29), пользуясь тем, что центр $M_{2}$ круга $V_{2}$ лежит в круге $V_{1}$, можно провести аналогичные рассуждения по отношению к кругу $V_{2}$ и таким образом доказать, что $\forall \mu>0$ при достаточно малых $\varepsilon$ сушествует $t_{2}=t_{2}(\mu, \varepsilon)>0$ такое, что

$$
\begin{array}{ll}
u(x, t, \varepsilon)<\varphi_{1}(x)+\mu / 2, & (x, t) \in \bar{V}_{2} \times\left[t_{2},+\infty\right), \\
u(x, t, \varepsilon)<\varphi_{2}(x)+\mu, & (x, t) \in \bar{D} \times\left[t_{2},+\infty\right) .
\end{array}
$$

Если $m>1$, то, используя тот же подход, устанавливаем соответствуюшие неравенства для $V_{3}$ и так далее до $V_{m+1}$ (если $m>2$ ). На последнем шаге мы приходим к выводу, что $\forall \mu>0$ при достаточно малых $\varepsilon$ сушествует $t_{m+1}=t_{m+1}(\mu, \varepsilon)>0$ такое, что справедливо неравенство

$$
u(x, t, \varepsilon)<\varphi_{1}(x)+\mu, \quad(x, t) \in \bar{V}_{m+1} \times\left[t_{m+1},+\infty\right) .
$$

Так как $V_{m+1}=E_{j}^{h / 4}$, то из (3.30) при $\mu=\eta$ следует $(3.23)$ с $T_{(j)}=t_{m+1}(\eta, \varepsilon)$.

Итак, доказано, что $\forall \eta>0$ и $\forall j \in \overline{1, n}$ при достаточно малых $\varepsilon$ существует $T_{(j)}>0$ такое, что для решения $u(x, t, \varepsilon)$ задачи (1.1)-(1.3) выполнено неравенство (3.23).

Так как число кругов $E_{i}^{h / 4}$ конечно, то из доказанного утверждения следует, что $\forall \eta>0$ при достаточно малых $\varepsilon$ вьполнено неравенство

$$
u(x, t, \varepsilon)<\varphi_{1}(x)+\eta, \quad(x, t) \in \bigcup_{i=1}^{n} \bar{E}_{i}^{h / 4} \times\left[T_{1},+\infty\right),
$$

с $T_{1}(\eta, \varepsilon)=\max _{1 \leqslant i \leqslant n} T_{(i)}>0$. Из (3.22) и (3.31) следует (3.19). Лемма доказана.

В следуюшей лемме содержится такое же утверждение, как в лемме 3.2, но для того случая, когда граница $D^{(-)}\left(D^{(+)}\right)$содержит границу $\partial D$ области $D$. 
ЛЕмма 3.3. Пусть выполнены условия (A1), (A4) и $D^{(-)}\left(D^{(+)}\right)$- область c гладкой границей $\partial D^{(-)}\left(\partial D^{(+)}\right)$такая, что $D^{(-)} \subseteq D\left(D^{(+)} \subseteq D\right), \quad \partial D \subseteq$ $\partial D^{(-)}\left(\partial D \subseteq \partial D^{(+)}\right) u$

$$
J(x)>0, \quad x \in \bar{D}^{(-)} \quad\left(J(x)<0, \quad x \in \bar{D}^{(+)}\right) .
$$

Пусть, далее, существует точка $x^{(-)} \in D^{(-)}\left(x^{(+)} \in D^{(+)}\right)$такая, что

$$
u_{0}\left(x^{(-)}\right)<0 \quad\left(u_{0}\left(x^{(+)}\right)>0\right) .
$$

Тогда $\forall \eta>0$ при достаточно мальх в существует $T_{2}=T_{2}(\eta, \varepsilon)>0$ такое, что решение $u(x, t, \varepsilon)$ задачи (1.1)-(1.3) удовлетворяет неравенствам

$$
\begin{aligned}
u(x, t, \varepsilon) & <\varphi_{1}(x)+\eta, & & (x, t) \in \bar{D}^{(-)} \times\left[T_{2},+\infty\right) \\
(u(x, t, \varepsilon) & >\varphi_{2}(x)-\eta, & & \left.(x, t) \in \bar{D}^{(+)} \times\left[T_{2},+\infty\right)\right) .
\end{aligned}
$$

ДокаЗАТЕЛЬСтво. Докажем первое утверждение леммы, относящееся к неравенству (3.32). Утверждение, относящееся к неравенству (3.33), доказывается аналогично.

Будем использовать локальные координаты $(z, \ell)$ в малой $\delta$-окрестности $\partial D$, введенные в $\S 2$.

Пусть число $z_{1} \in(0, \delta / 2]$ таково, что область, ограниченная кривой, имеющей в локальных координатах $(z, \ell)$ уравнение $z=z_{1}$, и кривой $\partial D$, принадлежит $D^{(-)}$, и пусть $z_{0}=z_{1} / 2$. Обозначим через $D_{\mathrm{ex}}^{(-)}$область, ограниченную кривой с уравнением $z=z_{0}$ и кривой $\partial D$, и введем область

$$
D_{\text {in }}^{(-)}=D^{(-)} \backslash D_{\text {ex }}^{(-)} .
$$

Уменьшим, если потребуется, $z_{1}$ так, чтобы

$$
\exists \bar{x}^{(-)} \in D_{\text {in }}^{(-)}: \quad u_{0}\left(\bar{x}^{(-)}\right)<0 .
$$

Введем обозначения: $f(u, z, \ell, \varepsilon) \equiv f(u, x(z, \ell), \varepsilon) ; \varphi_{i}(z, \ell) \equiv \varphi_{i}(x(z, \ell)), \quad i=$ 1,$2 ; u(z, \ell, t, \varepsilon) \equiv u(x(z, \ell), t, \varepsilon)$, где $u(x, t, \varepsilon)$ - решение задачи (1.1)-(1.3).

Определим функцию

$$
\beta(z, \ell, \mu, \nu)=\varphi_{1}(z, \ell)+\mu+Q_{0}(\tau, \ell, \nu)+\mu Q_{1}(\tau, \ell, \nu), \quad z \in\left[\nu, z_{1}\right], \quad \ell \in\left[0, \ell_{0}\right],
$$

где $\mu>0$ - число, которое уточним ниже, $\nu \in\left[0, z_{0}\right], \tau=(z-\nu) / \varepsilon$.

Функция $Q_{0}(\tau, \ell, \nu)$ является решением задачи ( $\nu$ играет роль параметра, $\nu \in$ $\left.\left[0, z_{0}\right]\right)$ :

$$
\begin{aligned}
\frac{\partial^{2}}{\partial \tau^{2}} Q_{0} & =f\left(\varphi_{1}(\nu, \ell)+Q_{0}, \nu, \ell, 0\right), \quad \tau>0, \\
Q_{0}(0, \ell, \nu) & =\varphi_{2}(\nu, \ell)-\varphi_{1}(\nu, \ell), \quad Q_{0}(+\infty, \ell, \nu)=0 .
\end{aligned}
$$

Число $z_{0}$ выбрано таким образом, что $J(x(\nu, \ell))>0$ при $\nu \in\left[0, z_{0}\right], \ell \in\left[0, \ell_{0}\right]$, поэтому $Q_{0}$ определяется однозначно, является положительной монотонно убывающей функцией $\tau$ при $\tau \geqslant 0$ и имеет экспоненциальные оценки по переменной $\tau$ сверху и снизу типа (3.5), (3.6), причем эти оценки равномерны по $\nu \in\left[0, z_{0}\right]$ и $\ell \in\left[0, \ell_{0}\right]$. 
Функцию $Q_{1}(\tau, \ell, \nu)$ определим как решение задачи

$$
\begin{aligned}
& \frac{\partial^{2}}{\partial \tau^{2}} Q_{1}=f_{u}(\tau, \ell, \nu) Q_{1}+f_{1}(\tau, \ell, \nu), \quad \tau>0 \\
& Q_{1}(0, \ell, \nu)=0, \quad Q_{1}(+\infty, \ell, \nu)=0
\end{aligned}
$$

где $f_{u}(\tau, \ell, \nu)=f_{u}\left(\varphi_{1}(\nu, \ell)+Q_{0}, \nu, \ell, 0\right), f_{1}(\tau, \ell, \nu)=\left[f_{u}(\tau, \ell, \nu)-f_{u}\left(\varphi_{1}(\nu, \ell)\right.\right.$, $\nu, \ell, 0)]$.

Функция $Q_{1}$ допускает явное представление и имеет равномерную по $\nu \in\left[0, z_{0}\right]$ и $\ell \in\left[0, \ell_{0}\right]$ экспоненциальную оценку типа (3.5).

По самому построению функция $\beta(z, \ell, \mu, \nu)$ удовлетворяет соотношению

$\varepsilon^{2} \Delta \beta-f(\beta, z, \ell, \varepsilon)=-\mu \bar{f}_{u}(z, \ell)+O(\varepsilon)+O\left(\mu^{2}\right), \quad z \in\left[\nu, z_{1}\right], \quad \ell \in\left[0, \ell_{0}\right], \quad \nu \in\left[0, z_{0}\right]$,

где $\bar{f}_{u}(z, \ell) \equiv f_{u}\left(\varphi_{1}(z, \ell), z, \ell, 0\right)$, откуда следует, что существует постоянная $C_{0}>0$, не зависящая от $\varepsilon, \mu, \nu$ и такая, что для любого достаточно малого $\mu$ при достаточно малых $\varepsilon$ выполняется неравенство

$$
\varepsilon^{2} \Delta \beta-f(\beta, z, \ell, \varepsilon)<-C_{0} \mu, \quad z \in\left[\nu, z_{1}\right], \quad \ell \in\left[0, \ell_{0}\right], \quad \nu \in\left[0, z_{0}\right] .
$$

Используя экспоненщиальные оценки функций $Q_{i}$, нетрудно доказать, что для любого достаточно малого $\mu$ при достаточно малых $\varepsilon$ имеет место неравенство

$$
\beta(z, \ell, \mu, \nu)>\varphi_{1}(z, \ell)+\mu / 2, \quad z \in\left[\nu, z_{1}\right], \quad \ell \in\left[0, \ell_{0}\right], \quad \nu \in\left[0, z_{0}\right] .
$$

Отметим также очевидное равенство

$$
\left.\beta(z, \ell, \mu, \nu)\right|_{z=\nu}=\varphi_{2}(\nu, \ell)+\mu, \quad \ell \in\left[0, \ell_{0}\right], \quad \nu \in\left[0, z_{0}\right]
$$

В силу (3.35) и того, что $D_{\text {in }}^{(-)} \subset D^{(-)}$(см. (3.34)), на основании леммы 3.2 и теоремы 2.1 можно заключить, что $\forall \mu>0$ при достаточно малых $\varepsilon$ существует $T_{(0)}=T_{(0)}(\mu, \varepsilon)>0$ такое, что решение $u(x, t, \varepsilon)$ задачи (1.1)-(1.3) удовлетворяет неравенствам

$$
\begin{array}{rlrl}
u(x, t, \varepsilon) & <\varphi_{1}(x)+\frac{\mu}{2}, & x & \in \bar{D}_{\text {in }}^{(-)}, \\
u(x, t, \varepsilon) & <\varphi_{2}(x)+\mu, & \in\left[T_{(0)},+\infty\right), \\
u, t) & \in \bar{D}, \quad t \in\left[T_{(0)},+\infty\right) .
\end{array}
$$

Введем функцию

$$
\begin{gathered}
w(z, \ell, t, \mu, \varepsilon)=[\beta(z, \ell, \mu, \nu(t))-u(z, \ell, t, \varepsilon)] \exp \left(-A\left(t-T_{(0)}\right)\right), \\
z \in\left[\nu(t), z_{1}\right], \quad \ell \in\left[0, \ell_{0}\right], \quad t \in\left[T_{(0)}, T_{(1)}\right]
\end{gathered}
$$

где

$$
\nu(t)=z_{0} \frac{T_{(1)}-t}{T_{(1)}-T_{(0)}},
$$

а величины $A$ и $T_{(1)}=T_{(1)}(\mu, \varepsilon)$ уточним ниже. Очевидно, что

$$
0 \leqslant \nu(t) \leqslant z_{0} \quad \text { при } t \in\left[T_{(0)}, T_{(1)}\right], \quad \nu\left(T_{(0)}\right)=z_{0}, \quad \nu\left(T_{(1)}\right)=0 .
$$


Используя (3.38), нетрудно получить неравенство

$$
\begin{gathered}
\varepsilon^{2} \Delta w-w_{t}-B w<\left(-C_{0} \mu-\frac{\partial \beta}{\partial \nu} \cdot \frac{z_{0}}{T_{(1)}-T_{(0)}}\right) \exp \left(-A\left(t-T_{(0)}\right)\right) \\
z \in\left[\nu(t), z_{1}\right], \quad \ell \in\left[0, \ell_{0}\right], \quad t \in\left[T_{(0)}, T_{(1)}\right]
\end{gathered}
$$

где $B=B(z, \ell, t, \varepsilon, \mu)=A+\int_{0}^{1} f_{u}(u+(\beta-u) s, z, \ell, \varepsilon) d s$ и, следовательно, $B>0$, если взять достаточно большое $A>0$.

Из вида $\beta(z, \ell, \mu, \nu)$ следует, что $\frac{\partial \beta}{\partial \nu}$ имеет оценку

$$
\left|\frac{\partial \beta}{\partial \nu}\right| \leqslant \frac{C_{1}}{\varepsilon},
$$

где постоянная $C_{1}>0$ не зависит от $\varepsilon, \nu, \mu$ (для ограниченного интервала изменения $\mu$ ). Поэтому, если взять $T_{(1)}=T_{(1)}(\mu, \varepsilon)$ такое, что

$$
T_{(1)}>T_{(0)}+\frac{C_{1} z_{0}}{C_{0} \mu \varepsilon}
$$

то для любого достаточно малого $\mu$ при достаточно малых $\varepsilon$ будет выполнено неравенство

$$
\varepsilon^{2} \Delta w-w_{t}-B w<0, \quad z \in\left[\nu(t), z_{1}\right], \quad \ell \in\left[0, \ell_{0}\right], \quad t \in\left[T_{(0)}, T_{(1)}\right] .
$$

Из (3.39) и (3.41) имеем

$$
\begin{array}{rrr}
w\left(z, \ell, T_{(0)}, \mu, \varepsilon\right)>0, & z \in\left[\nu\left(T_{(0)}\right), z_{1}\right], & \ell \in\left[0, \ell_{0}\right], \\
\left.w(z, \ell, t, \mu, \varepsilon)\right|_{z=z_{1}}>0, & t \in\left[T_{(0)}, T_{(1)}\right], & \ell \in\left[0, \ell_{0}\right],
\end{array}
$$

а из (3.40) и (3.42) получаем

$$
\left.w(z, \ell, t, \mu, \varepsilon)\right|_{z=\nu(t)}>0, \quad \ell \in\left[0, \ell_{0}\right], \quad t \in\left[T_{(0)}, T_{(1)}\right] .
$$

Из (3.43)-(3.46) очевидным образом следует, что

$$
w(z, \ell, t, \mu, \varepsilon)>0, \quad z \in\left[\nu(t), z_{1}\right], \quad \ell \in\left[0, \ell_{0}\right], \quad t \in\left[T_{(0)}, T_{(1)}\right],
$$

в частности, при $t=T_{(1)}$ имеем неравенство

$$
w\left(z, \ell, T_{(1)}, \mu, \varepsilon\right)>0, \quad z \in\left[\nu\left(T_{(1)}\right), z_{1}\right]=\left[0, z_{1}\right], \quad \ell \in\left[0, \ell_{0}\right],
$$

откуда получаем

$$
u\left(z, \ell, T_{(1)}, \varepsilon\right)<\beta\left(z, \ell, \mu, \nu\left(T_{(1)}\right)\right)=\beta(z, \ell, \mu, 0), \quad z \in\left[0, z_{1}\right], \quad \ell \in\left[0, \ell_{0}\right] .
$$

Возьмем произвольное $\eta>0$ и положим

$$
\eta_{0}=\min \left\{\eta, \min _{\left[0, \ell_{0}\right]}\left(\varphi_{2}(0, \ell)-\varphi_{1}(0, \ell)\right)\right\} .
$$


Введем функцию

$$
\tilde{\beta}(z, \ell, \mu, \gamma)=\varphi_{1}(z, \ell)+\mu+\widetilde{Q}_{0}(\tau, \ell, \gamma)+\mu \widetilde{Q}_{1}(\tau, \ell, \gamma), \quad z \in\left[0, z_{1}\right], \quad \ell \in\left[0, \ell_{0}\right]
$$

где $\tau=z / \varepsilon, \gamma \in[0,1]$, число $\mu>0$ - то же, что и в выражении для $\beta(z, \ell, \mu, \nu)$, а функции $\widetilde{Q}_{i}(\tau, \ell, \gamma)$ определим следуюшим образом.

Функция $\widetilde{Q}_{0}(\tau, \ell, \gamma)$ является решением задачи ( $\gamma$ играет роль параметра, $\gamma \in$ $[0,1])$ :

$$
\begin{aligned}
\frac{\partial^{2}}{\partial \tau^{2}} \widetilde{Q}_{0} & =f\left(\varphi_{1}(0, \ell)+\widetilde{Q}_{0}, 0, \ell, 0\right), \quad \tau>0 \\
\widetilde{Q}_{0}(0, \ell, \gamma) & =\varphi_{2}(0, \ell)-\varphi_{1}(0, \ell)-\gamma \psi(\ell), \quad \widetilde{Q}_{0}(+\infty, \ell, \gamma)=0
\end{aligned}
$$

где $\psi(\ell)=\varphi_{2}(0, \ell)-\varphi_{1}(0, \ell)-\eta_{0} / 2$. При любом $\gamma \in[0,1]$ функция $\widetilde{Q}_{0}(\tau, \ell, \gamma)$ положительна, монотонно убывает при $\tau \geqslant 0$ и имеет равномерные по $\gamma \in[0,1]$ и $\ell \in\left[0, \ell_{0}\right]$ экспоненщиальные оценки сверху и снизу типа $(3.5),(3.6)$. Из (3.49) следует, что

$$
\frac{\partial \widetilde{Q}_{0}}{\partial \tau}(0, \ell, \gamma)=-\left(2 \int_{\varphi_{1}(0, \ell)}^{\varphi_{2}(0, \ell)-\gamma \psi(\ell)} f(u, 0, \ell, 0) d u\right)^{\frac{1}{2}} \equiv-\sqrt{2} J_{\gamma}(\ell)
$$

Так как по условию леммы

$$
J_{0}(\ell)=(J(x(0, \ell)))^{\frac{1}{2}}=\left(\int_{\varphi_{1}(0, \ell)}^{\varphi_{2}(0, \ell)} f(u, 0, \ell, 0) d u\right)^{\frac{1}{2}}>0
$$

выполнено $(\mathrm{A} 1)$ и $\varphi_{2}(0, \ell)-\gamma \psi(\ell)>\varphi_{1}(0, \ell)$, то $J_{\gamma}(\ell)>0$ при $\ell \in\left[0, \ell_{0}\right], \gamma \in[0,1]$, и поэтому

$$
\frac{\partial \widetilde{Q}_{0}}{\partial \tau}(0, \ell, \gamma) \leqslant-C_{2}<0, \quad \ell \in\left[0, \ell_{0}\right], \quad \gamma \in[0,1]
$$

где $C_{2}=C_{2}\left(\eta_{0}\right)>0-$ некоторая постоянная.

Заметим, что

$$
\widetilde{Q}_{0}(\tau, \ell, 0)=Q_{0}(\tau, \ell, 0)
$$

где $Q_{0}(\tau, \ell, 0)$ - решение задачи $(3.36)$ при $\nu=0$.

Функция $\widetilde{Q}_{1}(\tau, \ell, \gamma)$ определяется аналогично функции $Q_{1}(\tau, \ell, \nu)($ см. $(3.37))$ как решение задачи

$$
\begin{aligned}
& \frac{\partial^{2}}{\partial \tau^{2}} \widetilde{Q}_{1}=\tilde{f}_{u}(\tau, \ell, \gamma) \widetilde{Q}_{1}+\tilde{f}_{1}(\tau, \ell, \gamma), \quad \tau>0, \\
& \widetilde{Q}_{1}(0, \ell, \gamma)=0, \quad \widetilde{Q}_{1}(+\infty, \ell, \gamma)=0,
\end{aligned}
$$

где $\tilde{f}_{u}(\tau, \ell, \gamma)=f_{u}\left(\varphi_{1}(0, \ell)+\widetilde{Q}_{0}, 0, \ell, 0\right), \tilde{f}_{1}(\tau, \ell, \gamma)=\left[\tilde{f}_{u}(\tau, \ell, \gamma)-f_{u}\left(\varphi_{1}(0, \ell)\right.\right.$, $0, \ell, 0)]$.

Отметим, что

$$
\widetilde{Q}_{1}(\tau, \ell, 0)=Q_{1}(\tau, \ell, 0)
$$


В силу (3.52) и $(3.53) \tilde{\beta}(z, \ell, \mu, 0)=\beta(z, \ell, \mu, 0)$, и поэтому неравенство $(3.47)$ можно переписать в виде

$$
u\left(z, \ell, T_{(1)}, \varepsilon\right)<\tilde{\beta}(z, \ell, \mu, 0), \quad z \in\left[0, z_{1}\right], \quad \ell \in\left[0, \ell_{0}\right] .
$$

Отметим также, что при достаточно малых $\varepsilon$ имеет место очевидное неравенство

$$
\left.\tilde{\beta}(z, \ell, \mu, \gamma)\right|_{z=z_{1}}>\varphi_{1}\left(z_{1}, \ell\right)+\frac{\mu}{2}, \quad \ell \in\left[0, \ell_{0}\right], \quad \gamma \in[0,1] .
$$

Из (3.51) следует, что для любого достаточно малого $\mu$ при достаточно малых $\varepsilon$ $\tilde{\beta}_{z}(0, \ell, \mu, \gamma)=\frac{1}{\varepsilon}\left[\frac{\partial \widetilde{Q}_{0}}{\partial \tau}(0, \ell, \gamma)+\mu \frac{\partial \widetilde{Q}_{1}}{\partial \tau}(0, \ell, \gamma)\right]+O(1)<0, \quad \ell \in\left[0, \ell_{0}\right], \quad \gamma \in[0,1]$.

Кроме того, по самому построению функции $\tilde{\beta}(z, \ell, \mu, \gamma)$ для любого достаточно малого $\mu$ при достаточно малых $\varepsilon$ имеет место неравенство вида (3.38):

$$
\varepsilon^{2} \Delta \tilde{\beta}-f(\tilde{\beta}, z, \ell, \varepsilon)<-\widetilde{C}_{0} \mu, \quad z \in\left[0, z_{1}\right], \quad \ell \in\left[0, \ell_{0}\right], \quad \gamma \in[0,1],
$$

где $\widetilde{C}_{0}>0$ - не зависяшая от $\varepsilon, \mu$ и $\gamma$ постоянная.

Введем функцию

$$
\begin{aligned}
\widetilde{w}(z, \ell, t, \mu, \varepsilon) & =[\tilde{\beta}(z, \ell, \mu, \gamma(t))-u(x, t, \varepsilon)] \exp \left(-\tilde{A}\left(t-T_{(1)}\right)\right), \\
z & \in\left[0, z_{1}\right], \quad \ell \in\left[0, \ell_{0}\right], \quad t \in\left[T_{(1)}, T_{(2)}\right]
\end{aligned}
$$

где

$$
\gamma(t)=\frac{t-T_{(1)}}{T_{(2)}-T_{(1)}}
$$

а величины $\tilde{A}$ и $T_{(2)}$ уточнены ниже. Заметим, что $\gamma\left(T_{(1)}\right)=0, \gamma\left(T_{(2)}\right)=1$.

Используя (3.57), получаем неравенство

$$
\begin{gathered}
\varepsilon^{2} \Delta \widetilde{w}-\widetilde{w}_{t}-\widetilde{B} \widetilde{w}<\left(-\widetilde{C}_{0} \mu-\frac{\partial \tilde{\beta}}{\partial \gamma} \cdot \frac{1}{T_{(2)}-T_{(1)}}\right) \exp \left(-\tilde{A}\left(t-T_{(1)}\right)\right), \\
z \in\left[0, z_{1}\right], \quad \ell \in\left[0, \ell_{0}\right], \quad t \in\left[T_{(1)}, T_{(2)}\right],
\end{gathered}
$$

где $\widetilde{B}=\tilde{A}+\int_{0}^{1} f_{u}(u+(\tilde{\beta}-u) s, x, \varepsilon) d s>0$ при достаточно большом $\tilde{A}>0$.

Производная $\frac{\partial \tilde{\beta}}{\partial \gamma}$ имеет оценку $\left|\frac{\partial \widetilde{\beta}}{\partial \gamma}\right| \leqslant \widetilde{C}_{1}$, где $\widetilde{C}_{1}>0$-постоянная, не зависящая от $\varepsilon, \gamma$ и $\mu$ (для ограниченного интервала изменения $\mu$ ). Поэтому если взять $T_{(2)}=$ $T_{(2)}(\mu, \varepsilon)$ такое, что

$$
T_{(2)}>T_{(1)}+\frac{\widetilde{C}_{1}}{\widetilde{C}_{0} \mu},
$$

то будет выполнено неравенство

$$
\varepsilon^{2} \Delta \widetilde{w}-\widetilde{w}_{t}-\widetilde{B} \widetilde{w}<0, \quad z \in\left[0, z_{1}\right], \quad \ell \in\left[0, \ell_{0}\right], \quad t \in\left[T_{(1)}, T_{(2)}\right] .
$$

Из (3.54) имеем неравенство

$$
\widetilde{w}\left(z, \ell, T_{(1)}, \mu, \varepsilon\right)>0, \quad z \in\left[0, z_{1}\right], \quad \ell \in\left[0, \ell_{0}\right],
$$


а из (3.56) и (1.2) получаем

$$
\widetilde{w}_{z}(0, \ell, t, \mu, \varepsilon)<0, \quad t \in\left[T_{(1)}, T_{(2)}\right], \quad \ell \in\left[0, \ell_{0}\right] .
$$

Наконец, (3.41) и (3.55) дают неравенство

$$
\widetilde{w}\left(z_{1}, \ell, t, \mu, \varepsilon\right)>0, \quad t \in\left[T_{(1)}, T_{(2)}\right], \quad \ell \in\left[0, \ell_{0}\right] .
$$

Из (3.58)-(3.61) очевидным образом следует, что $\widetilde{w}(z, \ell, t, \mu, \varepsilon)>0, z \in\left[0, z_{1}\right]$, $\ell \in\left[0, \ell_{0}\right], t \in\left[T_{(1)}, T_{(2)}\right]$, в частности, $\widetilde{w}\left(z, \ell, T_{(2)}, \mu, \varepsilon\right)>0, z \in\left[0, z_{1}\right], \ell \in\left[0, \ell_{0}\right]$, откуда получаем

$$
u\left(z, \ell, T_{(2)}, \varepsilon\right)<\tilde{\beta}\left(z, \ell, \mu, \gamma\left(T_{(2)}\right)\right)=\tilde{\beta}(z, \ell, \mu, 1), \quad z \in\left[0, z_{1}\right], \quad \ell \in\left[0, \ell_{0}\right] .
$$

Из (3.41) и (3.55) следует, что

$$
u\left(z_{1}, \ell, t, \varepsilon\right)<\tilde{\beta}\left(z_{1}, \ell, \mu, 1\right), \quad \ell \in\left[0, \ell_{0}\right], \quad t \in\left[T_{(2)},+\infty\right) .
$$

Неравенства (3.62), (3.63) и неравенства $(3.56),(3.57)$, взятые при $\gamma=1$, показывают, что для любого достаточно малого $\mu$ при достаточно малых $\varepsilon$ функция $\tilde{\beta}(z, \ell, \mu, 1)$ является стационарным верхним барьером для решения $u(z, \ell, t, \varepsilon)$ при $z \in\left[0, z_{1}\right], \ell \in\left[0, \ell_{0}\right], t \in\left[T_{(2)},+\infty\right)$, т.e.

$$
u(z, \ell, t, \varepsilon)<\tilde{\beta}(z, \ell, \mu, 1), \quad z \in\left[0, z_{1}\right], \quad \ell \in\left[0, \ell_{0}\right], \quad t \in\left[T_{(2)},+\infty\right) .
$$

Поскольку $0 \leqslant \widetilde{Q}_{0}(\tau, \ell, 1) \leqslant \eta_{0} / 2 \leqslant \eta / 2$, то для любого достаточно малого $\mu$ при достаточно малых $\varepsilon$ будет выполнено неравенство

$$
\tilde{\beta}(z, \ell, \mu, 1)<\varphi_{1}(z, \ell)+\eta, \quad z \in\left[0, z_{1}\right], \quad \ell \in\left[0, \ell_{0}\right] .
$$

Из (3.64), (3.65) следует, что

$$
u(x, t, \varepsilon)<\varphi_{1}(x)+\eta, \quad x \in \bar{D}_{\mathrm{ex}}^{(-)}, \quad t \in\left[T_{(2)},+\infty\right) .
$$

Зафиксируем столь малое $\mu \in[0, \eta]$, чтобы при достаточно малых $\varepsilon$ выполнялись неравенства (3.41) и (3.66). Тогда из (3.41) и (3.66) в силу (3.34) получим неравенство (3.32) с $T_{2}=T_{(2)}$. Очевидно, что $T_{2}=T_{2}(\eta, \varepsilon)>0$. Лемма доказана.

В следуюшей лемме показано, что решение задачи (1.1)-(1.3) удовлетворяет неравенствам (3.32), (3.33) и при иных условиях, нежели в лемме 3.3 .

ЛЕмма 3.4. Пусть выполнены условия (A1), (A4), и пусть $\Omega^{(-)}\left(\Omega^{(+)}\right)$область с гладкой граничей $\partial \Omega^{(-)}\left(\partial \Omega^{(+)}\right)$такая, что:

1) $\Omega^{(-)} \subseteq D\left(\Omega^{(+)} \subseteq D\right)$ и либо $\bar{\Omega}^{(-)} \cap \partial D=\varnothing\left(\bar{\Omega}^{(+)} \cap \partial D=\varnothing\right)$, либо $\partial D \subseteq \partial \Omega^{(-)}\left(\partial D \subseteq \partial \Omega^{(+)}\right)$;

2) $u_{0}(x)<0, \quad x \in \bar{\Omega}^{(-)}\left(u_{0}(x)>0, x \in \bar{\Omega}^{(+)}\right)$;

3) $J(x)>0, \quad x \in \partial \Omega^{(-)} \backslash \partial D\left(J(x)<0, \quad x \in \partial \Omega^{(+)} \backslash \partial D\right)$.

Тогда для любого $\eta>0$ существует $T_{3}=T_{3}(\eta)>0$ такое, ито при достаточно малых в решение $u(x, t, \varepsilon)$ задачи (1.1)-(1.3) удовлетворяет неравенству

$$
\begin{aligned}
u(x, t, \varepsilon)<\varphi_{1}(x)+\eta, & & (x, t) \in \bar{\Omega}^{(-)} \times\left[T_{3},+\infty\right) \\
\left(u(x, t, \varepsilon)>\varphi_{2}(x)-\eta,\right. & & \left.(x, t) \in \bar{\Omega}^{(+)} \times\left[T_{3},+\infty\right)\right) .
\end{aligned}
$$


ДоказАТЕльСтво. Докажем утверждение, относящееся к неравенству (3.67). Утверждение, относяшееся к неравенству (3.68), доказывается аналогично.

Рассмотрим сначала случай, когда $\bar{\Omega}^{(-)} \cap \partial D=\varnothing$.

Через $\partial \Omega_{1}^{(-)} \subset \partial \Omega^{(-)}$обозначим ту (замкнутую) кривую, для которой область $\Omega^{(-)}$является внутренней. Если $\Omega^{(-)}-$многосвязная область, то через $\partial \Omega_{i}^{(-)}$, $i=2, \ldots, n$, обозначим те (замкнутые) кривые, входящие в $\partial \Omega^{(-)}$, для которых область $\Omega^{(-)}$является внешней.

Для точек $M$ из малой окрестности кривой $\partial \Omega_{1}^{(-)}$введем локальные координаты $(z, \ell)$, где $|z|$ - расстояние от $M$ до $\partial \Omega_{1}^{(-)}$вдоль нормали к $\partial \Omega_{1}^{(-)}, z=|z|$, если $M$ принадлежит внутренней по отношению к $\partial \Omega_{1}^{(-)}$подобласти области $D$, $z=-|z|$, если - внешней подобласти, и $z=0$, если $M \in \partial \Omega_{1}^{(-)}$, а $\ell$ - координата той точки на $\partial \Omega_{1}^{(-)}$, из которой вьпушена указанная нормаль, $\ell \in\left[0, \ell_{0}\right]$, где $\ell_{0}-$ некоторое число.

Будем считать, что число $\delta>0$ таково, что при $|z| \leqslant \delta$ соответствие между координатами $(z, \ell)$ и $x=\left(x_{1}, x_{2}\right)$ взаимно однозначно, а число $d \in(0, \delta / 2]$ выберем так, что

$$
\begin{aligned}
J(x(z, \ell))>0, & z=-d, & \ell \in\left[0, \ell_{0}\right], \\
u_{0}(x(z, \ell))<0, & z \in[-d, 0], & \ell \in\left[0, \ell_{0}\right],
\end{aligned}
$$

и кривая $\partial \Omega_{1}$, имеющая в локальных координатах $(z, \ell)$ уравнение $z=-d$, целиком лежит в $D$.

Пусть $\varphi_{i}(z, \ell) \equiv \varphi_{i}(x(z, \ell)), f(u, z, \ell, \varepsilon) \equiv f(u, x(z, \ell), \varepsilon)$.

Определим функцию $Q_{0}(\tau, \ell,-d), \tau=(z+d) / \varepsilon$, как решение задачи $(3.36)$ (см. доказательство леммы 3.3) при $\nu=-d$.

Аналогично тому, как это было сделано при доказательстве леммы 3.3 , введем обозначения $f_{u}(\tau, \ell,-d), f_{1}(\tau, \ell,-d)$ и определим функцию $Q_{1}(\tau, \ell,-d)$ как решение задачи $(3.37)$ при $\nu=-d$. Положим

$$
\stackrel{(1)}{Q}=\left[Q_{0}(\tau, \ell,-d)+\mu Q_{1}(\tau, \ell,-d)\right] \zeta\left(\frac{z+d}{d}\right), \quad \tau \geqslant 0,
$$

где $\zeta$ - срезающая функция, введенная в $\S 2$.

Если область $\Omega^{(-)}$многосвязна, то для каждой кривой $\partial \Omega_{i}^{(-)}, i \in \overline{2, n}$, аналогичным образом введем (свои) локальные координаты $(z, \ell)$ и соответствующее число $\delta_{i}>0$ и выберем число $d_{i} \in\left[0, \delta_{i} / 2\right]$ так, что выполнено неравенство из (3.69) при $z=d_{i}, \quad \ell \in\left[0, \ell_{0}\right]$, а неравенство из (3.70) - при $z \in\left[0, d_{i}\right]$. Через $\partial \Omega_{i}$ обозначим кривую, имеющую в локальных координатах $(z, \ell)$ уравнение $z=d_{i}$.

Далее, так же, как и выше, используя аналогичные обозначения, определим функции $Q_{0}\left(\tau, \ell, d_{i}\right), \tau=\left(z-d_{i}\right) / \varepsilon$, и $Q_{1}\left(\tau, \ell, d_{i}\right)$ как решения задач $(3.36)$ и $(3.37)$ при $\nu=d_{i}$ и положим

$$
\stackrel{(i)}{Q}=\left[Q_{0}\left(-\tau, \ell, d_{i}\right)+\mu Q_{1}\left(-\tau, \ell, d_{i}\right)\right] \zeta\left(\frac{d_{i}-z}{d_{i}}\right), \quad \tau \leqslant 0 .
$$

Пусть $\Omega$ - область с гранищей $\partial \Omega=\bigcup_{i=1}^{n} \partial \Omega_{i}$. 
Ясно, что $u_{0}(x)<0, x \in \bar{\Omega}$, а значит, в силу теоремы 2.1 существует $T_{0}=$ $T_{0}(\mu / 2)>0$ такое, что при достаточно малых $\varepsilon$ решение $u(x, t, \varepsilon)$ задачи $(1.1)-(1.3)$ удовлетворяет неравенствам

$$
\begin{aligned}
u\left(x, T_{0}, \varepsilon\right) & <\varphi_{1}(x)+\mu / 2, & x & \in \bar{\Omega}, \\
u(x, t, \varepsilon) & <\varphi_{2}(x)+\mu, & (x, t) & \in \partial \Omega \times\left[T_{0},+\infty\right) .
\end{aligned}
$$

Введем функцию

$$
\beta(x, \mu)=\varphi_{1}(x)+\mu+\sum_{i=1}^{n} \stackrel{(i)}{Q}, \quad x \in \bar{\Omega} .
$$

Используя (3.71), (3.72), нетрудно проверить (это делается таким же образом, как при доказательстве леммы 3.3), что для любого достаточно малого $\mu$ при достаточно малых $\varepsilon$ функция $\beta(x, \mu)$ является стационарным верхним барьером для решения $u(x, t, \varepsilon)$ при $(x, t) \in \bar{\Omega} \times\left[T_{0},+\infty\right)$, т.е.

$$
u(x, t, \varepsilon)<\beta(x, \mu), \quad(x, t) \in \bar{\Omega} \times\left[T_{0},+\infty\right) .
$$

(i)

Учитывая наличие срезающих функций в выражениях для $Q$, можно утверждать, что $\beta(x, \mu)=\varphi_{1}(x)+\mu$ при $x \in \bar{\Omega}^{(-)}$, и поэтому из неравенства (3.73) при $\mu=\eta$ последует (3.67) с $T_{3}=T_{0}$.

Перейдем теперь к случаю, когда $\partial D \subseteq \partial \Omega^{(-)}$. В этом случае функцию $\beta(x, \mu)$ строим аналогичным образом, с тем отличием, что теперь $\partial \Omega_{1} \equiv \partial D$, а в качестве $(1)$ $Q$ возьмем функцию

$$
\stackrel{(1)}{Q}=\varepsilon \exp (-\kappa \tau) \zeta\left(\frac{z}{\delta}\right), \quad \tau \geqslant 0
$$

где $\tau=z / \varepsilon$ (локальные координаты $(z, \ell)$ в малой $\delta$-окрестности $\partial D$ были введены в $\S 2)$, а $\kappa>0$ - число, которое выбирается так, что

$$
\frac{\partial \beta}{\partial n}>0, \quad x \in \partial D
$$

$\frac{\partial}{\partial n}-$ производная по направлению внешней нормали к $\partial D$.

Как и выше, нетрудно доказать, что для любого достаточно малого $\mu$ при достаточно малых $\varepsilon$ функция $\beta(x, \mu)$ является стационарным верхним барьером для решения $u(x, t, \varepsilon)$ при $(x, t) \in \bar{\Omega} \times\left[T_{0},+\infty\right)$, откуда при $\mu=(2 / 3) \eta$ последует $(3.67)$ с $T_{3}=T_{0}$. Лемма доказана.

3.2. Основные результаты. Для точек $M\left(x_{1}, x_{2}\right)$ из малой окрестности кривой Г (см. условие (А2)) введем локальные координаты $(r, \theta)$, где $|r|$ - расстояние от точки $M$ до кривой $\Gamma$ вдоль нормали к $\Gamma$, которая проходит через $M, \theta \in\left[0, \theta_{0}\right]$ $\left(\theta_{0}\right.$ - некоторое число $)$ - координата той точки кривой $\Gamma$, из которой выпушена указанная нормаль, $r=|r|$, если $M \in D_{0}^{(+)}, r=-|r|$, если $M \in D_{0}^{(-)}, r=0$, если $M \in \Gamma$.

Пусть число $\delta_{0}>0$ таково, что при $|r| \leqslant 2 \delta_{0}$ соответствие между координатами $(r, \theta)$ и $\left(x_{1}, x_{2}\right)$ взаимно однозначно. 
ТЕОРема 3.1. Пусть выполнены условия (A1)-(А5).

Тогда $\forall \eta>0 u \forall d \in\left(0, \delta_{0}\right]$ при достаточно мальи $\varepsilon$ существует $T_{4}=$ $T_{4}(\eta, d, \varepsilon)>0$ такое, что решение $u(x, t, \varepsilon)$ задачи (1.1)-(1.3) удовлетворяет неравенствам

$$
\begin{aligned}
u(x, t, \varepsilon) & <\varphi_{1}(x)+\eta, & & (x, t) \in \overline{D_{0}^{(-)} \backslash D^{d}} \times\left[T_{4},+\infty\right),(3) \\
u(x, t, \varepsilon) & >\varphi_{2}(x)-\eta, & & (x, t) \in \overline{D_{0}^{(+)} \backslash D^{d}} \times\left[T_{4},+\infty\right), \\
\varphi_{1}(x)-\eta<u(x, t, \varepsilon) & <\varphi_{2}(x)+\eta, & & (x, t) \in \bar{D} \times\left[T_{4},+\infty\right),
\end{aligned}
$$

где $D^{d}=\{M(r, \theta) \in D:|r|<d\}$.

ДокАЗАТЕЛЬСтво. Докажем (3.74) (неравенство (3.75) доказывается аналогично, а неравенства (3.76) доказаны в теореме 2.1).

Пусть $d \in\left(0, \delta_{0}\right]$ столь мало, что

$$
x^{(-)} \in D_{0}^{(-)} \backslash \bar{D}^{d}, \quad J(x)>0, \quad x \in D_{0}^{(-)} \cap \bar{D}^{d},
$$

где $x^{(-)}$- точка из условия (А5), и $\bar{D}^{d} \cap \partial D=\varnothing$.

Возможны два случая:

1) $J(x)>0, x \in \overline{D_{0}^{(-)} \backslash D^{d}}$; в этом случае (3.74) следует из леммы 3.3 ;

2) $\exists x \in \overline{D_{0}^{(-)} \backslash D^{d}}: J(x) \leqslant 0$; в этом случае через $\Omega_{i}, i=1,2, \ldots, n$, обозначим элементы класса $N$ (см. § 1), принадлежашие $\overline{D_{0}^{(-)} \backslash D^{d}}$ (т.е. те точки, кривые и подобласти области $\overline{D_{0}^{(-)} \backslash D^{d}}$, где $\left.J(x) \leqslant 0\right)$.

В силу (А3) число таких элементов конечно.

Учитьвая требования (А3) и (А5), можно выбрать столь малое число $h>0$, что для каждого из пересечений $\Omega_{i}^{h} h$-окрестностей элементов $\Omega_{i}, i=1,2, \ldots, n$, с $D_{0}^{(-)}$будут выполнены следующие условия:

1) область $\Omega_{i}^{h}$ имеет гладкую границу $\partial \Omega_{i}^{h}$;

2) $\bar{\Omega}_{i}^{h} \cap \bar{\Omega}_{i^{\prime}}^{h}=\varnothing$ при $i \neq i^{\prime}, \bar{\Omega}_{i}^{h} \cap \bar{D}^{d}=\varnothing$ и либо $\partial D \subset \partial \Omega_{i}^{h}$, либо $\partial D \cup \bar{\Omega}_{i}^{h}=\varnothing$;

3) $J(x)>0, x \in \partial \Omega_{i}^{h} \backslash \partial D ; u_{0}(x)<0, x \in \bar{\Omega}_{i}^{h}$.

Отсюда согласно лемме 3.4 следует, что для любого $\eta>0$ существует такое $T^{\prime}=T^{\prime}(\eta)>0$, что при достаточно малых $\varepsilon$ неравенство из (3.74) выполняется для $(x, t) \in \bar{\Omega}_{i}^{h} \times\left[T^{\prime},+\infty\right), i=1,2, \ldots, n$.

Удалим теперь из области $\bar{D}_{0}^{(-)} \backslash \bar{D}^{d}$ все $\bar{\Omega}_{i}^{h}, i=1,2, \ldots, n$. Останутся области, которые обозначим через $D_{j}, j=1,2, \ldots, m$.

Очевидно, что при $j=1,2, \ldots, m$ справедливы следующие утверждения:

1) область $D_{j}$ имеет гладкую границу $\partial D_{j}$;

2) либо $\partial D \subset \partial D_{j}$, либо $\partial D \cup \bar{D}_{j}=\varnothing$;

3) $J(x)>0, x \in \bar{D}_{j}$, и на границе $\partial D_{j}$ имеются точки, в которых $u_{0}(x)<0$, а значит,

$$
\exists x_{j}^{(-)} \in D_{j}: \quad u_{0}\left(x_{j}^{(-)}\right)<0 .
$$

Отсюда следует, что $\forall j \in \overline{1, m}$ на области $D^{(-)}=D_{j}$ мы можем использовать либо лемму 3.2 , если $\bar{D}_{j} \cap \partial D=\varnothing$, либо лемму 3.3 , если $\partial D \subset \partial D_{j}$. Поэтому $\forall \eta>0$ при достаточно малых $\varepsilon$ существует такое $T^{\prime \prime}=T^{\prime \prime}(\eta, d, \varepsilon)>0$, что неравенство из (3.74) выполняется для $(x, t) \in \bar{D}_{j} \times\left[T^{\prime \prime},+\infty\right), j=1,2, \ldots, m$. 
Объединяя полученные в п. 2) результаты, получаем, что $\forall \eta>0$ при достаточно малых $\varepsilon$ сушествует $T_{4}=T_{4}(\eta, d, \varepsilon)>0$ такое, что выполнено неравенство (3.74). Теорема доказана.

Результат теоремы 3.1 можно усилить, заменив в (3.74)-(3.76) $\eta$ на величину порядка $\varepsilon^{n+1}$, а $T_{4}$ - на некоторое $T_{5}(n, d, \varepsilon)$. С этой целью рассмотрим упоминавшиеся в $\oint 1$ стационарные решения $u^{(1)}(x, \varepsilon)$ и $u^{(2)}(x, \varepsilon)$ задачи $(1.1),(1.2)$ без внутренних переходных слоев, близкие при малых $\varepsilon$ соответственно к функциям $\varphi_{1}(x)$ и $\varphi_{2}(x)$ во всей области $\bar{D}$. Используя алгоритм метода пограничных функций [3], для этих решений нетрудно построить и обосновать $\forall n \geqslant 0$ асимптотики $U_{n}^{(1)}(x, \varepsilon)$ и $U_{n}^{(2)}(x, \varepsilon)$, имеющие вид

$$
\begin{array}{ll}
U_{n}^{(1)}(x, \varepsilon)=\bar{u}_{n}^{(-)}(x, \varepsilon)+P_{n+1}^{(-)}(\xi, \ell, \varepsilon), & x \in \bar{D} \\
U_{n}^{(2)}(x, \varepsilon)=\bar{u}_{n}^{(+)}(x, \varepsilon)+P_{n+1}^{(+)}(\xi, \ell, \varepsilon), & x \in \bar{D},
\end{array}
$$

где

$$
\begin{gathered}
\bar{u}_{n}^{(-)}(x, \varepsilon)=\varphi_{1}(x)+\sum_{i=1}^{n} \varepsilon^{i} \bar{u}_{i}^{(-)}(x), \\
\bar{u}_{n}^{(+)}(x, \varepsilon)=\varphi_{2}(x)+\sum_{i=1}^{n} \varepsilon^{i} \bar{u}_{i}^{(+)}(x), \\
P_{n+1}^{( \pm)}(\xi, \ell, \varepsilon)=\sum_{i=1}^{n+1} \varepsilon^{i} P_{i}^{( \pm)}(\xi, \ell), \\
\xi=-\frac{z}{\varepsilon}
\end{gathered}
$$

локальные координаты $(z, \ell)$ в окрестности $\partial D$ были введены в $\oint 2$. Функции $\bar{u}_{i}^{( \pm)}$ (регулярные члены асимптотики) определяются как решения линейных алгебраических уравнений, например: $\bar{u}_{1}^{(-)}(x)=-f_{\varepsilon}\left(\varphi_{1}(x), x, 0\right) / f_{u}\left(\varphi_{1}(x), x, 0\right)$; функции $P_{i}^{( \pm)}(\xi, \ell)$ являются решениями линейных дифференциальных уравнений с постоянными коэффициентами при $\xi<0$, они имеют экспоненциальные оценки вида $\left|P_{1}^{( \pm)}(\xi, \ell)\right| \leqslant C \exp (m \xi)$, равномерные по $\ell \in\left[0, \ell_{0}\right]$, а при $\xi=0$ удовлетворяющие условиям

$$
\frac{\partial P_{i}^{( \pm)}}{\partial \xi}(0, \ell)=\frac{\partial \bar{u}_{i-1}^{( \pm)}}{\partial z}(0, \ell)
$$

$\left(\bar{u}_{i-1}^{( \pm)}(z, \ell) \equiv \bar{u}_{i-1}^{( \pm)}(x(z, \ell))\right)$, откуда следует, что $U_{n}^{(1)}$ и $U_{n}^{(2)}$ удовлетворяют граничным условиям (1.2). Будем считать, что функции $P_{i}^{( \pm)}$умножены на срезающие функции.

Для удобства результат о существовании и асимптотике решений $u^{(1)}(x, \varepsilon)$ и $u^{(2)}(x, \varepsilon)$ задачи $(1.1),(1.2)$ сформулируем в виде леммы.

Лемма 3.5. Пусть выполнено условие (A1). Тогда при достаточно мальх в существуют стационарные решения $u^{(1)}(x, \varepsilon)$ и $u^{(2)}(x, \varepsilon)$ из $C^{2}(\bar{D})$ задачи (1.1), (1.2) такие, что $\forall n \geqslant 0$

$$
\begin{aligned}
& \left\|u^{(1)}(x, \varepsilon)-U_{n}^{(1)}(x, \varepsilon)\right\|_{C(\bar{D})}=O\left(\varepsilon^{n+1}\right), \\
& \left\|u^{(2)}(x, \varepsilon)-U_{n}^{(2)}(x, \varepsilon)\right\|_{C(\bar{D})}=O\left(\varepsilon^{n+1}\right) .
\end{aligned}
$$


СЛЕДСТВИЕ 3.1. В неравенствах (3.74)-(3.76) можно заменить $\varphi_{1}(x)$ u $\varphi_{2}(x)$ на $u^{(1)}(x, \varepsilon)$ и $u^{(2)}(x, \varepsilon)$ соответственно.

Усилением результатов теорем 2.1 и 3.1 является следующая теорема.

ТЕОРема 3.2. Пусть выполнены условия (A1)-(А5).

Тогда $\forall n \geqslant 0$ u $\forall d \in\left(0, \delta_{0}\right]$ при достаточно малых в существуют $T_{5}=$ $T_{5}(n, d, \varepsilon)>0$ и число $\bar{C}_{n}>0$, не зависящее от $\varepsilon$, такие, что решение $u(x, t, \varepsilon)$ задачи (1.1)-(1.3) удовлетворяет неравенствам

$$
\begin{aligned}
& u(x, t, \varepsilon)<u^{(1)}(x, \varepsilon)+\bar{C}_{n} \varepsilon^{n+1}, \quad(x, t) \in \overline{D_{0}^{(-)} \backslash D^{d}} \times\left[T_{5},+\infty\right), \\
& u(x, t, \varepsilon)>u^{(2)}(x, \varepsilon)-\bar{C}_{n} \varepsilon^{n+1}, \quad(x, t) \in \overline{D_{0}^{(+)} \backslash D^{d}} \times\left[T_{5},+\infty\right), \\
& u^{(1)}(x, \varepsilon)-\bar{C}_{n} \varepsilon^{n+1}<u(x, t, \varepsilon)<u^{(2)}(x, \varepsilon)+\bar{C}_{n} \varepsilon^{n+1}, \quad(x, t) \in \bar{D} \times\left[T_{5},+\infty\right),
\end{aligned}
$$

зде $D^{d}=\{M(r, \theta) \in D:|r|<d\}$.

ДокАЗАтЕЛЬСтво. Пусть $d \in\left(0, \delta_{0}\right]$ фиксировано так, что $\bar{D}^{d} \cap \partial D=\varnothing$ и $J(x)>0, x \in D_{0}^{(-)} \cap \bar{D}^{d}$.

Докажем неравенство (3.80). Положим $\bar{r}=-d / 2$ и введем область $D^{\bar{r}}=$ $\{M(r, \theta) \in D: \bar{r}<r \leqslant 0\}$. Через $\Gamma_{\bar{r}}$ будем обозначать кривую, имеющую в локальных координатах $(r, \theta)$ уравнение $r=\bar{r}$. Определим $\forall n \geqslant 0$ функцию $\beta_{n}(x, \varepsilon)$ следуюшим образом:

$$
\begin{aligned}
\beta_{n}(x, \varepsilon)= & \beta_{n}^{(-)}(x, \varepsilon)=\bar{u}_{n+1}^{(-)}(x, \varepsilon)+\varepsilon^{n+1}+P_{n+2}^{(-)}(\xi, \ell, \varepsilon)+Q_{n+1}^{(-)}(\tau, \theta, \varepsilon) \\
& +\varepsilon^{n+1} q_{\beta}^{(-)}(\tau, \theta), \quad x \in \overline{D_{0}^{(-)} \backslash D^{\bar{r}}}, \\
\beta_{n}(x, \varepsilon)= & \beta_{n}^{(+)}(x, \varepsilon)+v_{n \beta}(r, \theta, \varepsilon)=\bar{u}_{n+1}^{(+)}(x, \varepsilon)+\varepsilon^{n+1}+Q_{n+1}^{(+)}(\tau, \theta, \varepsilon) \\
& +\varepsilon^{n+1} q_{\beta}^{(+)}(\tau, \theta)+v_{n \beta}(r, \theta, \varepsilon), \quad x \in \overline{D_{0}^{(+)} \cup D^{\bar{r}}},
\end{aligned}
$$

где $\bar{u}_{n+1}^{( \pm)}(x, \varepsilon), P_{n+2}^{(-)}(\xi, \ell, \varepsilon)$ определены формулами $(3.77),(3.78)$,

$$
Q_{n+1}^{( \pm)}(\tau, \theta, \varepsilon)=\sum_{i=0}^{n+1} \varepsilon^{i} Q_{i}^{( \pm)}(\tau, \theta), \quad \tau=\frac{r-\bar{r}}{\varepsilon}
$$

$Q_{i}^{(-)}(\tau, \theta)$ и $Q_{i}^{(+)}(\tau, \theta)$ - погранслойные функции из асимптотических разложений таких решений краевых задач

$$
\begin{aligned}
& \varepsilon^{2} \Delta u^{(-)}=f\left(u^{(-)}, x, \varepsilon\right), \quad x \in D_{0}^{(-)} \backslash D^{\bar{r}}, \\
& \left.\frac{\partial u^{(-)}}{\partial n}\right|_{\partial D}=0,\left.\quad u^{(-)}\right|_{\Gamma_{\bar{r}}}=0,
\end{aligned}
$$

и

$$
\varepsilon^{2} \Delta u^{(+)}=f\left(u^{(+)}, x, \varepsilon\right), \quad x \in D_{0}^{(+)} \cup D^{\bar{r}},\left.\quad u^{(+)}\right|_{\Gamma_{\bar{r}}}=0,
$$

которые близки при малых $\varepsilon$ соответственно к $\varphi_{1}(x)$ на $\bar{D}_{0}^{(-)} \backslash \bar{D}^{\bar{r}}$ и к $\varphi_{2}(x)$ на $D_{0}^{(+)} \cup D^{\bar{r}}$. Функции $Q_{i}^{( \pm)}(\tau, \theta)$ нетрудно построить, используя алгоритм метода пограничных функций [3]. 
Функция $Q_{0}^{(-)}$определяется как решение задачи

$$
\begin{aligned}
& \frac{\partial^{2}}{\partial \tau^{2}} Q_{0}^{(-)}=f\left(\varphi_{1}(\bar{r}, \theta)+Q_{0}^{(-)}, \bar{r}, \theta, 0\right), \quad \tau<0 \\
& Q_{0}^{(-)}(0, \theta)=-\varphi_{1}(\bar{r}, \theta), \quad Q_{0}^{(-)}(-\infty, \theta)=0
\end{aligned}
$$

где использованы обозначения $f(u, r, \theta, \varepsilon) \equiv f(u, x(r, \theta), \varepsilon), \varphi_{i}(r, \theta) \equiv \varphi_{i}(x(r, \theta))$, а функция $Q_{0}^{(+)}$определяется как решение аналогичной задачи

$$
\begin{aligned}
& \frac{\partial^{2}}{\partial \tau^{2}} Q_{0}^{(+)}=f\left(\varphi_{2}(\bar{r}, \theta)+Q_{0}^{(+)}, \bar{r}, \theta, 0\right), \quad \tau>0 \\
& Q_{0}^{(+)}(0, \theta)=-\varphi_{2}(\bar{r}, \theta), \quad Q_{0}^{(+)}(+\infty, \theta)=0 .
\end{aligned}
$$

Функции $Q_{0}^{( \pm)}$монотонно возрастают и

$$
\begin{aligned}
& \frac{\partial Q_{0}^{(-)}}{\partial \tau}(0, \theta)=\left(2 \int_{\varphi_{1}(\bar{r}, \theta)}^{0} f(u, \bar{r}, \theta, 0) d u\right)^{1 / 2} \\
& \frac{\partial Q_{0}^{(+)}}{\partial \tau}(0, \theta)=\left(2 \int_{\varphi_{2}(\bar{r}, \theta)}^{0} f(u, \bar{r}, \theta, 0) d u\right)^{1 / 2} .
\end{aligned}
$$

Формулы (3.83) получаются так же, как (3.50).

При $i \geqslant 1$ функции $Q_{i}^{( \pm)}(\tau, \theta)$ являются решениями линейных задач. Отметим, что $Q_{i}^{( \pm)}(\tau, \theta)$ имеют равномерные по $\theta \in\left[0, \theta_{0}\right]$ экспоненщиальные оценки сверху по переменной $\tau$ типа $(3.5)$, а $Q_{0}^{( \pm)}(\tau, \theta)$ - еще и оценки снизу типа (3.6).

Функции $q_{\beta}^{(-)}, q_{\beta}^{(+)}$- погранслойные добавки, появляющиеся при построении верхних решений указанных краевых задач методом, описанным в [10]. Например, функция $q_{\beta}^{(-)}(\tau, \theta)$ определяется как решение задачи

$$
\begin{aligned}
& \frac{\partial^{2}}{\partial \tau^{2}} q_{\beta}^{(-)}=f_{u}^{(-)}(\tau, \theta) q_{\beta}^{(-)}+f_{u}^{(-)}(\tau, \theta)-\bar{f}_{u}^{(-)}(\theta)-\Psi^{(-)}(\tau), \quad \tau<0, \\
& q_{\beta}^{(-)}(0, \theta)=0, \quad q_{\beta}^{(-)}(-\infty, \theta)=0,
\end{aligned}
$$

где $f_{u}^{(-)}(\tau, \theta) \equiv f_{u}\left(\varphi_{1}(\bar{r}, \theta)+Q_{0}^{(-)}(\tau, \theta), \bar{r}, \theta, 0\right), \bar{f}_{u}^{(-)}(\theta) \equiv f_{u}\left(\varphi_{1}(\bar{r}, \theta), \bar{r}, \theta, 0\right)$, a $\Psi^{(-)}(\tau)>0$ - функция, которая имеет экспоненциальную оценку и выбирается так, что при достаточно малых $\varepsilon$ выполняется неравенство

$$
\beta_{n}^{(-)}(x, \varepsilon)-\beta_{n+1}^{(-)}(x, \varepsilon) \geqslant m_{n} \varepsilon^{n+1}, \quad x \in \overline{D_{0}^{(-)} \backslash D^{\bar{r}}},
$$

$m_{n}$ - положительное число, не зависящее от $\varepsilon$.

Умножим функции $Q_{i}^{( \pm)}, q_{\beta}^{( \pm)}, P_{i}^{(-)}$на соответствующие срезающие функции, не изменяя при этом их обозначений, и определим функцию

$$
v_{n \beta}=\left.\left[\beta_{n}^{(-)}(x, \varepsilon)-\beta_{n}^{(+)}(x, \varepsilon)\right]\right|_{\Gamma_{\bar{r}}} \zeta((r-\bar{r}) / d) ;
$$

функция $\zeta$ определена в $\S 2$. 
Функция $v_{n \beta}$ обеспечивает непрерывность $\beta_{n}(x, \varepsilon)$ при $x \in \Gamma_{\bar{r}}$. Заметим, что $v_{n \beta}=O\left(\varepsilon^{n+2}\right)$, а на кривой $\Gamma_{\bar{r}}$ при достаточно малых $\varepsilon$ справедливо неравенство

$$
\left.\varepsilon\left[\frac{\partial}{\partial r} \beta_{n}^{(-)}-\frac{\partial}{\partial r} \beta_{n}^{(+)}\right]\right|_{\Gamma_{\bar{r}}} \geqslant\left. C_{0} J(x)\right|_{\Gamma_{\bar{r}}}+O(\varepsilon)>0
$$

где $C_{0}>0$ - некоторое число. Неравенство (3.85) нетрудно доказать, используя формулы (3.83).

Из самого способа построения функции $\beta_{n}(x, \varepsilon)$ при достаточно малых $\varepsilon$ следуют соотношения (см. также [10])

$$
\begin{aligned}
& \varepsilon^{2} \Delta \beta_{n}-f\left(\beta_{n}, x, \varepsilon\right)=-\left[f_{u}\left(\varphi_{1}(x), x, 0\right)+\Psi^{(-)}(\tau)\right] \varepsilon^{n+1}+O\left(\varepsilon^{n+2}\right)<0 \\
& x \in \overline{D_{0}^{(-)} \backslash D^{\bar{r}}} \\
& \varepsilon^{2} \Delta \beta_{n}-f\left(\beta_{n}, x, \varepsilon\right)=-\left[f_{u}\left(\varphi_{2}(x), x, 0\right)+\Psi^{(+)}(\tau)\right] \varepsilon^{n+1}+O\left(\varepsilon^{n+2}\right)<0 \\
& x \in D_{0}^{(+)} \cup D^{\bar{r}} \\
&\left.\frac{\partial \beta_{n}}{\partial n}\right|_{\partial D}=0
\end{aligned}
$$

где $\Psi^{(+)}(\tau)>0$ - функция, аналогичная упомянутой выше функции $\Psi^{(-)}(\tau)$.

Зафиксируем $n \geqslant 0$ и введем функцию

$$
\hat{v}(x, t, \varepsilon)=\beta_{n}(x, \varepsilon)+\frac{\mu}{\varepsilon} \Theta(x, \varepsilon) \exp (-\mu t)
$$

где $\Theta(x, \varepsilon)=\beta_{0}(x, \varepsilon)-\beta_{1}(x, \varepsilon)$, причем $\mu>0$ выберем настолько малым (но фиксированным при $\varepsilon \rightarrow 0$ ), что при достаточно малых $\varepsilon$ будут выполнены неравенства

$$
\begin{gathered}
\varepsilon^{2} \Delta \hat{v}-\hat{v}_{t}-f(\hat{v}, x, \varepsilon)<0, \quad(x, t) \in D \times(0,+\infty), \\
\left.\frac{\partial \hat{v}}{\partial r}\right|_{r=\bar{r}-0} \geqslant\left.\frac{\partial \hat{v}}{\partial r}\right|_{r=\bar{r}+0}, \quad t \in[0,+\infty)
\end{gathered}
$$

(на кривой $\Gamma_{\bar{r}}$ неравенство (3.89) будет выполнено для предельных значений левой части (3.89) с одной и с другой стороны от $\left.\Gamma_{\bar{r}}\right)$. Покажем для $(3.89)$, что такой выбор $\mu$ возможен. Раскладывая $f(\hat{v}, x, \varepsilon)$ по формуле Тейлора с центром разложения $\left(\beta_{n}, x, \varepsilon\right)$, получаем

$$
\begin{aligned}
\varepsilon^{2} \Delta \hat{v}- & \hat{v}_{t}-f(\hat{v}, x, \varepsilon)=\left\{\varepsilon^{2} \Delta \beta_{n}-f\left(\beta_{n}, x, \varepsilon\right)\right\} \\
+ & \left\{\frac{\mu}{\varepsilon}\left[\varepsilon^{2} \Delta \Theta-f_{u}\left(\beta_{n}, x, \varepsilon\right) \Theta\right] \exp (-\mu t)\right\} \\
+ & \left\{-\frac{\mu^{2}}{\varepsilon} \Theta\left[-1+\frac{1}{2} f_{u u}^{*} \cdot \frac{\Theta}{\varepsilon} \exp (-\mu t)\right] \exp (-\mu t)\right\} .
\end{aligned}
$$

Первое из трех слагаемых, заключенных в фигурные скобки в правой части (3.91), при достаточно малых $\varepsilon$ отрицательно в силу (3.86), (3.87). Третье слагаемое в силу очевидной оценки $\Theta(x, \varepsilon)=O(\varepsilon)$ при достаточно мальх $\varepsilon$ не превосходит по абсолютной величине функции $C_{1} \mu^{2} \exp (-\mu t)$, где $C_{1}>0$ не зависит от $\varepsilon$ и $\mu$. 
Оценим функцию $\varepsilon^{2} \Delta \Theta-f_{u}\left(\beta_{n}, x, \varepsilon\right) \Theta$, входящую во второе слагаемое в правой части (3.91). Вычитая из равенств (3.86), (3.87) при $n=0$ эти же равенства при $n=1$ и учитьвая, что $\left[f_{u}\left(\varphi_{i}(x), x, 0\right)+\Psi^{( \pm)}(\tau)\right] \geqslant C_{2}>0$, получаем

$$
\varepsilon^{2} \Delta \Theta-\left[f\left(\beta_{0}, x, \varepsilon\right)-f\left(\beta_{1}, x, \varepsilon\right)\right] \leqslant-C_{2} \varepsilon+O\left(\varepsilon^{2}\right) .
$$

Так как $\forall n \geqslant 0$

$$
f\left(\beta_{0}, x, \varepsilon\right)-f\left(\beta_{1}, x, \varepsilon\right)=f_{u}\left(\beta_{1}, x, \varepsilon\right) \Theta+\frac{1}{2} f_{u u}^{*} \cdot \Theta^{2}=f_{u}\left(\beta_{n}, x, \varepsilon\right) \Theta+O\left(\varepsilon^{2}\right),
$$

то из (3.92) при достаточно малых $\varepsilon$ следует неравенство

$$
\varepsilon^{2} \Delta \Theta-f_{u}\left(\beta_{n}, x, \varepsilon\right) \Theta \leqslant-C_{2} \varepsilon+O\left(\varepsilon^{2}\right) \leqslant-C_{3} \varepsilon,
$$

где $C_{3}>0$ не зависит от $\varepsilon$. Вследствие этого второе слагаемое в правой части (3.91) при достаточно малых $\varepsilon$ не превосходит отрицательной функции $-C_{3} \mu \exp (-\mu t)$, и, таким образом, мы приходим к неравенству

$$
\varepsilon^{2} \Delta \hat{v}-\hat{v}_{t}-f(\hat{v}, x, \varepsilon) \leqslant\left[-C_{3} \mu+C_{1} \mu^{2}\right] \exp (-\mu t)
$$

откуда следует, что для любого достаточно малого $\mu$ при достаточно малых $\varepsilon$ выполняется (3.89). Неравенство (3.90) будет выполнено для любого достаточно малого $\mu$ при достаточно малых $\varepsilon$ в силу (3.85).

Далее, в силу (3.88) имеем

$$
\left.\frac{\partial \hat{v}}{\partial n}\right|_{\partial D}=0, \quad t \in[0,+\infty)
$$

Из (3.84) при $n=0$ и аналогичного неравенства для области $\overline{D_{0}^{(+)} \cup D^{\bar{r}}}$ имеем

$$
\beta_{0}(x, \varepsilon)-\beta_{1}(x, \varepsilon)=\Theta(x, \varepsilon) \geqslant m_{0} \varepsilon .
$$

Отсюда и из неубывания функций $Q_{0}^{( \pm)}(\tau, \theta)$ следует, что существует постоянная $C_{4}>0$ такая, что при достаточно малых $\varepsilon$

$$
\hat{v}(x, 0, \varepsilon)>\varphi_{1}(x)+C_{4}, \quad x \in \bar{D} .
$$

В силу экспоненциальной оценки $Q$-функций и неравенства $\Theta(x, \varepsilon) \geqslant m_{0} \varepsilon$ существует постоянная $C_{5}>0$ такая, что при достаточно малых $\varepsilon$

$$
\hat{v}(x, 0, \varepsilon)>\varphi_{2}(x)+C_{5}, \quad x \in \overline{D_{0}^{(+)} \cup D^{\bar{r} / 2}},
$$

где $D^{\bar{r} / 2}=\{M(r, \theta) \in D: \bar{r} / 2<r \leqslant 0\}$.

Применяя теорему 3.1 для $\eta=\min \left\{C_{4}, C_{5}\right\}$ и $d=|\bar{r}| / 2$, получаем, что при достаточно малых $\varepsilon$ существует $T_{4}>0$ такое, что (см. (3.74), (3.76))

$$
\begin{array}{ll}
u\left(x, T_{4}, \varepsilon\right)<\varphi_{1}(x)+C_{4}, & x \in \overline{D_{0}^{(-)} \backslash D^{\bar{r} / 2}}, \\
u\left(x, T_{4}, \varepsilon\right)<\varphi_{2}(x)+C_{5}, & x \in \bar{D} .
\end{array}
$$


Отсюда и из $(3.94),(3.95)$ имеем неравенство

$$
u\left(x, T_{4}, \varepsilon\right)<\hat{v}(x, 0, \varepsilon), \quad x \in \bar{D} .
$$

Соотношения (3.89), (3.90), (3.93) и (3.96) показывают, что функция $\hat{v}\left(x, t-T_{4}, \varepsilon\right)$ является (негладким) верхним барьером для решения $u(x, t, \varepsilon)$ задачи $(1.1)-(1.3)$ при $t \geqslant T_{4}$, т.е.

$$
u(x, t, \varepsilon)<\hat{v}\left(x, t-T_{4}, \varepsilon\right), \quad(x, t) \in \bar{D} \times\left[T_{4},+\infty\right) .
$$

Но в силу экспоненциальной оценки функций $Q_{i}^{(-)}$и $q_{\beta}^{(-)}$, выбора $\bar{r}$, а также леммы 3.5 существует постоянная $\bar{C}_{n}>0$ такая, что при достаточно малых $\varepsilon$

$$
\hat{v}(x, t, \varepsilon)<u^{(1)}(x, \varepsilon)+\bar{C}_{n} \varepsilon^{n+1}, \quad(x, t) \in \overline{D_{0}^{(-)} \backslash D^{d}} \times[1 / \varepsilon,+\infty) .
$$

Отсюда и из (3.97) следует, что

$$
u(x, t, \varepsilon)<u^{(1)}(x, \varepsilon)+\bar{C}_{n} \varepsilon^{n+1}, \quad(x, t) \in \overline{D_{0}^{(-)} \backslash D^{d}} \times\left[T_{4}+1 / \varepsilon,+\infty\right),
$$

т.е. неравенство (3.80) выполняется для $T_{5}=T_{4}+1 / \varepsilon>0$.

Доказательство неравенства (3.81) проводится аналогично.

Неравенства (3.82) можно доказать при помоши верхнего $\hat{v}(x, t)$ и нижнего $\bar{v}(x, t)$ решений задачи (1.1), (1.2) следуюшего вида:

$$
\hat{v}(x, t)=u^{(2)}(x, \varepsilon)+\mu \exp (-\mu t), \quad \bar{v}(x, t)=u^{(1)}(x, \varepsilon)-\mu \exp (-\mu t),
$$

где $\mu>0$ - достаточно малое, но фиксированное при $\varepsilon \rightarrow 0$ число. При этом надо воспользоваться следствием 3.1. Теорема доказана.

\section{$\S 4$. Предельный переход к стационарному решению при $t \rightarrow \infty$}

Пусть выполнены условия (A1)-(А5).

4.1. Построение асимптотики специального вида. Пусть $d \in\left(0, \delta_{0}\right]$ выбрано так, что $\bar{D}^{d} \cap \partial D=\varnothing$ и

$$
J(x)>0, \quad x \in \bar{D}^{d} \cap D_{0}^{(-)} ; \quad J(x)<0, \quad x \in \bar{D}^{d} \cap D_{0}^{(+)} ;
$$

число $\delta_{0}>0$ и множество $D^{d}-$ те же, что в $\S 3$.

Рассмотрим функцию

$$
\begin{array}{ll}
U(x, \tau, \gamma, \varepsilon)=U^{(-)}(x, \gamma, \varepsilon)=\bar{u}_{3}^{(-)}(x, \varepsilon)+\sum_{i=0}^{3} \varepsilon^{i} \widetilde{Q}_{i}^{(-)}(\tau, \theta, \gamma), & x \in \bar{\Omega}_{\gamma}^{(-)}, \\
U(x, \tau, \gamma, \varepsilon)=U^{(+)}(x, \gamma, \varepsilon)=\bar{u}_{3}^{(+)}(x, \varepsilon)+\sum_{i=0}^{3} \varepsilon^{i} \widetilde{Q}_{i}^{(+)}(\tau, \theta, \gamma), & x \in \bar{\Omega}_{\gamma}^{(+)}
\end{array}
$$

где $\bar{u}_{3}^{( \pm)}(x, \varepsilon)$ определены формулами (3.77),

$$
\tau=\frac{r-r_{\gamma}}{\varepsilon}, \quad r_{\gamma}=\lambda_{0}(\gamma)+\sum_{i=1}^{3} \varepsilon^{i} \lambda_{i}(\gamma, \theta), \quad \lambda_{0}(\gamma)=-\gamma,
$$


функции $\widetilde{Q}_{i}^{( \pm)}$и $\lambda_{i}$ определим ниже, $\Omega_{\gamma}^{(+)}$-внутренняя, а $\Omega_{\gamma}^{(-)}$-внешняя подобласти области $D$ по отношению к кривой $\Lambda_{\gamma}$, имеюшей в локальных координатах $(r, \theta)$ (см. $\S 3$ ) уравнение $r=r_{\gamma}, \gamma \in\left[-\gamma_{0}, \gamma_{0}\right]$. Значение $\gamma_{0}$ мы уточним ниже, а пока будем считать, что $\gamma_{0}<d$.

Заметим, что оператор $\varepsilon^{2} \Delta$ имеет в координатах $(r, \theta)$ следующий вид:

$$
\varepsilon^{2} \Delta=\varepsilon^{2}\left(\frac{\partial^{2}}{\partial r^{2}}+a_{1}(r, \theta) \frac{\partial^{2}}{\partial \theta^{2}}+a_{2}(r, \theta) \frac{\partial}{\partial r}+a_{3}(r, \theta) \frac{\partial}{\partial \theta}\right),
$$

где $a_{1}(r, \theta), a_{2}(r, \theta), a_{3}(r, \theta)$ - известные функции [2]-[4].

Используя алгоритм метода пограничных функций [3], определим $\widetilde{Q}_{i}^{( \pm)}, i=\overline{0,3}$, и $\lambda_{i}(\gamma, \theta), i=1,2,3$, так, чтобы равенства

$$
\begin{gathered}
\varepsilon^{2} \Delta U^{(-)}-f\left(U^{(-)}, x, \varepsilon\right)=0, \quad x \in \bar{\Omega}_{\gamma}^{(-)}, \quad \gamma \in\left[-\gamma_{0}, \gamma_{0}\right], \\
\varepsilon^{2} \Delta U^{(+)}-f\left(U^{(+)}, x, \varepsilon\right)=0, \quad x \in \bar{\Omega}_{\gamma}^{(+)}, \quad \gamma \in\left[-\gamma_{0}, \gamma_{0}\right], \\
\left.U^{(-)}\right|_{\Lambda_{\gamma}}=\left.U^{(+)}\right|_{\Lambda_{\gamma}}=0, \quad \gamma \in\left[-\gamma_{0}, \gamma_{0}\right],
\end{gathered}
$$

выполнялись с точностью $O\left(\varepsilon^{4}\right)$, т.е. во всех приближениях по $\varepsilon$ от нулевого до третьего порядка, а равенство

$$
\left.\varepsilon \frac{\partial U^{(-)}}{\partial r}\right|_{\Lambda_{\gamma}}=\left.\varepsilon \frac{\partial U^{(+)}}{\partial r}\right|_{\Lambda_{\gamma}}, \quad \gamma \in\left[-\gamma_{0}, \gamma_{0}\right]
$$

выполнялось во всех приближениях по $\varepsilon$ до третьего порядка включительно, кроме, может быть, нулевого приближения.

Для функций $\widetilde{Q}_{0}^{( \pm)}(\tau, \theta, \gamma)$ получаются такие же задачи, как и для функций $Q_{0}^{( \pm)}(\tau, \theta)$ в доказательстве теоремы 3.2, но с той разницей, что в задаче для $Q_{0}^{(-)}$ и аналогичной задаче для $Q_{0}^{(+)}$нужно заменить $\bar{r}$ на $\lambda_{0}(\gamma)$. Поэтому для $\frac{\partial \widetilde{Q}_{0}^{( \pm)}}{\partial \tau}(0, \theta, \gamma)$ имеют место выражения типа (3.83) и мы получаем равенство

$$
\begin{aligned}
\frac{\partial \widetilde{Q}_{0}^{(-)}}{\partial \tau}(0, \theta, \gamma)-\frac{\partial \widetilde{Q}_{0}^{(+)}}{\partial \tau}(0, \theta, \gamma)= & \sqrt{2}\left[\left(\int_{\varphi_{1}\left(\lambda_{0}(\gamma), \theta\right)}^{0} f\left(u, \lambda_{0}(\gamma), \theta, 0\right) d u\right)^{\frac{1}{2}}\right. \\
& \left.-\left(\int_{\varphi_{2}\left(\lambda_{0}(\gamma), \theta\right)}^{0} f\left(u, \lambda_{0}(\gamma), \theta, 0\right) d u\right)^{\frac{1}{2}}\right],
\end{aligned}
$$

из которого при указанных ограничениях на $\gamma_{0}\left(\gamma_{0}<d\right)$ в силу $(4.1)$ следуют неравенства

$$
\begin{aligned}
& \frac{\partial \widetilde{Q}_{0}^{(-)}}{\partial \tau}(0, \theta, \gamma)-\frac{\partial \widetilde{Q}_{0}^{(+)}}{\partial \tau}(0, \theta, \gamma)>0 \text { при } \gamma \in\left(0, \gamma_{0}\right], \\
& \frac{\partial \widetilde{Q}_{0}^{(-)}}{\partial \tau}(0, \theta, \gamma)-\frac{\partial \widetilde{Q}_{0}^{(+)}}{\partial \tau}(0, \theta, \gamma)<0 \text { при } \gamma \in\left[-\gamma_{0}, 0\right) .
\end{aligned}
$$

Тем самым равенство (4.4) в нулевом приближении по $\varepsilon$, т.е. равенство

$$
\frac{\partial \widetilde{Q}_{0}^{(-)}}{\partial \tau}(0, \theta, \gamma)=\frac{\partial \widetilde{Q}_{0}^{(+)}}{\partial \tau}(0, \theta, \gamma)
$$


вьполняется только при $\gamma=0$.

Для $\widetilde{Q}_{1}^{(-)}$имеем задачу

$$
\begin{gathered}
\frac{\partial^{2}}{\partial \tau^{2}} \widetilde{Q}_{1}^{(-)}=f_{u}^{(-)}(\tau, \theta, \gamma) \widetilde{Q}_{1}^{(-)}+f_{1}^{(-)}(\tau, \theta, \gamma), \quad \tau<0 \\
\widetilde{Q}_{1}^{(-)}(0, \theta, \gamma)=-\varphi_{1 r}\left(\lambda_{0}(\gamma), \theta\right) \lambda_{1}(\gamma, \theta)-\bar{u}_{1}^{(-)}\left(\lambda_{0}(\gamma), \theta\right), \quad \widetilde{Q}_{1}^{(-)}(-\infty, \theta, \gamma)=0
\end{gathered}
$$

где

$$
\begin{aligned}
f_{1}^{(-)}(\tau, \theta, \gamma)= & f_{u}^{(-)}(\tau, \theta, \gamma)\left[\bar{u}_{1}^{(-)}\left(\lambda_{0}(\gamma), \theta\right)+\varphi_{1 r}\left(\lambda_{0}(\gamma), \theta\right)\left(\tau+\lambda_{1}(\gamma, \theta)\right)\right] \\
& +f_{r}^{(-)}(\tau, \theta, \gamma)\left(\tau+\lambda_{1}(\gamma, \theta)\right)+f_{\varepsilon}^{(-)}(\tau, \theta, \gamma) \\
& -a_{2}\left(\lambda_{0}(\gamma), \theta\right) \frac{\partial \widetilde{Q}_{0}^{(-)}}{\partial \tau}(\tau, \theta, \gamma)
\end{aligned}
$$

функция $a_{2}$ здесь та же, что и в $(4.3)$, а обозначения $f_{u}^{(-)}(\tau, \theta, \gamma), f_{r}^{(-)}(\tau, \theta, \gamma)$ и $f_{\varepsilon}^{(-)}(\tau, \theta, \gamma)$ здесь использованы для соответствуюших производных функции $f(u, r, \theta, \varepsilon)=f(u, x(r, \theta), \varepsilon)$, вычисленных в точке $\left(\varphi_{1}\left(\lambda_{0}(\gamma), \theta\right)+\widetilde{Q}_{0}^{(-)}(\tau, \theta, \gamma)\right.$, $\left.\lambda_{0}(\gamma), \theta, 0\right)$. Аналогичная задача имеет место для $\widetilde{Q}_{1}^{(+)}(\tau, \theta, \gamma)$ при $\tau>0$.

Известно [4], что функции $\widetilde{Q}_{1}^{( \pm)}(\tau, \theta, \gamma)$ однозначно определяются из этих задач, выражаются в явном виде и зависят линейно от неизвестной пока функции $\lambda_{1}(\gamma, \theta)$.

Из равенства (4.4) в первом приближении по $\varepsilon$ получаем

$$
\frac{\partial \widetilde{Q}_{1}^{(-)}}{\partial \tau}(0, \theta, \gamma)+\varphi_{1 r}\left(\lambda_{0}(\gamma), \theta\right)=\frac{\partial \widetilde{Q}_{1}^{(+)}}{\partial \tau}(0, \theta, \gamma)+\varphi_{2 r}\left(\lambda_{0}(\gamma), \theta\right)
$$

Отсюда, используя явные выражения для $\widetilde{Q}_{1}^{( \pm)}(\tau, \theta, \gamma)$, приходим к равенству

$$
I(\gamma, \theta) \lambda_{1}(\gamma, \theta)=\Phi_{1}(\gamma, \theta)
$$

где

$$
\begin{aligned}
I(\gamma, \theta)= & {\left[\frac{\partial \widetilde{Q}_{0}^{(-)}}{\partial \tau}(0, \theta, \gamma)\right]^{-1} \int_{\varphi_{1}\left(\lambda_{0}(\gamma), \theta\right)}^{0} f_{r}\left(u, \lambda_{0}(\gamma), \theta, 0\right) d u } \\
& +\left[\frac{\partial \widetilde{Q}_{0}^{(+)}}{\partial \tau}(0, \theta, \gamma)\right]^{-1} \int_{0}^{\varphi_{2}\left(\lambda_{0}(\gamma), \theta\right)} f_{r}\left(u, \lambda_{0}(\gamma), \theta, 0\right) d u
\end{aligned}
$$

$\Phi_{1}(\gamma, \theta)$ - известная функция.

Заметим, что так как $\left.\frac{\partial J}{\partial r}\right|_{r=0}=\left.\frac{\partial J}{\partial n_{0}}\right|_{\Gamma}$, то в силу условия (А2)

$$
I(0, \theta)=\left.\left[\frac{\partial \widetilde{Q}_{0}^{(-)}}{\partial \tau}(0, \theta, 0)\right]^{-1} \frac{\partial J}{\partial r}\right|_{r=0}<0
$$

Возьмем $\gamma_{0}$ столь малым, чтобы выполнялось неравенство

$$
I(\gamma, \theta)<0, \quad \gamma \in\left[-\gamma_{0}, \gamma_{0}\right]
$$


Теперь можно выбрать $\lambda_{1}(\gamma, \theta)$ так, чтобы равенство (4.4) было выполнено в первом приближении по $\varepsilon$, т.е. чтобы было выполнено равенство (4.6). Для этого нужно положить

$$
\lambda_{1}(\gamma, \theta)=\frac{\Phi_{1}(\gamma, \theta)}{I(\gamma, \theta)} .
$$

Продолжая построение в соответствии с алгоритмом из [3], [4], определим $\widetilde{Q}_{2}^{( \pm)}$, $\lambda_{2}$ и $\widetilde{Q}_{3}^{( \pm)}, \lambda_{3}$, причем $\lambda_{i}(\gamma, \theta), i=2,3$, будут найдены из условия, что равенство (4.4) выполняется в $i$-ом приближении по $\varepsilon$.

Для $\lambda_{i}(\gamma, \theta), i=2,3$, получаются уравнения типа (4.6), однозначно разрешимые в силу неравенства (4.7).

Итак, функция $U(x, \tau, \gamma, \varepsilon)$ полностью построена, причем функции $\widetilde{Q}_{i}^{( \pm)}$имеют равномернье по $\gamma \in\left[-\gamma_{0}, \gamma_{0}\right]$ и $\theta \in\left[0, \theta_{0}\right]$ экспоненциальные оценки сверху по переменной $\tau$ типа (3.5), а $\widetilde{Q}_{0}^{( \pm)}$имеют еше и оценки снизу типа (3.6).

4.2. Модификация асимптотики. Построим функцию $\tilde{\beta}(x, \gamma, \varepsilon)$ в виде

$$
\begin{aligned}
\tilde{\beta}(x, \gamma, \varepsilon)= & \tilde{\beta}^{(-)}(x, \gamma, \varepsilon)=U\left(x, \tau_{\beta}, \gamma, \varepsilon\right)+\varepsilon^{3}\left(1+Q^{(-)}\left(\tau_{\beta}, \theta, \gamma\right)\right) \\
& +P_{4}^{(-)}(\xi, \ell, \varepsilon), \quad x \in \bar{\Omega}_{\gamma, \beta}^{(-)}, \\
\tilde{\beta}(x, \gamma, \varepsilon)= & \tilde{\beta}^{(+)}(x, \gamma, \varepsilon)=U\left(x, \tau_{\beta}, \gamma, \varepsilon\right)+\varepsilon^{3}\left(1+Q^{(+)}\left(\tau_{\beta}, \theta, \gamma\right)\right) \\
& +v_{\beta}(r, \theta, \varepsilon), \quad x \in \bar{\Omega}_{\gamma, \beta}^{(+)},
\end{aligned}
$$

где

$$
\tau_{\beta}=\left(r-r_{\beta}\right) / \varepsilon, \quad r_{\beta}=r_{\gamma}-\varepsilon^{3} \sigma,
$$

$\sigma>0, \Omega_{\gamma, \beta}^{(-)}-$внешняя, а $\Omega_{\gamma, \beta}^{(+)}$- внутренняя подобласти области $D$ по отношению к кривой $\Lambda_{\gamma, \beta}$, имеющей в локальных координатах $(r, \theta)$ уравнение $r=r_{\beta}, \gamma \in$ $\left[0, \gamma_{0}\right]$.

Функции $U(x, \tau, \gamma, \varepsilon)$ и $r_{\gamma}$ определены в п. 4.1, причем $U=U^{(-)}$в $(4.8)$ и $U=$ $U^{(+)}$в $(4.9)$, функция $P_{4}^{(-)}(\xi, \ell, \varepsilon)$ определена формулой $(3.78)$, функция $v_{\beta}$ будет выбрана ниже. Остановимся на построении функций $Q^{( \pm)}$и выборе $\sigma>0$.

Считая, что $\gamma \in\left[-\gamma_{0}, \gamma_{0}\right]$, определим функции $Q^{( \pm)}(\tau, \theta, \gamma)$ как решения следующих задач:

$$
\begin{aligned}
\frac{\partial^{2}}{\partial \tau^{2}} Q^{(-)}= & f_{u}^{(-)}(\tau, \theta, \gamma) Q^{(-)}+f_{u}^{(-)}(\tau, \theta, \gamma)\left(1-\sigma \varphi_{1 r}\left(\lambda_{0}(\gamma), \theta\right)\right) \\
& -f_{r}^{(-)}(\tau, \theta, \gamma) \sigma-f_{u}^{(-)}(\gamma, \theta), \quad \tau<0 \\
Q^{(-)}(0, \theta, \gamma)= & \varphi_{1 r}\left(\lambda_{0}(\gamma), \theta\right) \sigma, \quad Q^{(-)}(-\infty, \theta, \gamma)=0 \\
\frac{\partial^{2}}{\partial \tau^{2}} Q^{(+)}= & f_{u}^{(+)}(\tau, \theta, \gamma) Q^{(+)}+f_{u}^{(+)}(\tau, \theta, \gamma)\left(1-\sigma \varphi_{2 r}\left(\lambda_{0}(\gamma), \theta\right)\right) \\
& -f_{r}^{(+)}(\tau, \theta, \gamma) \sigma-f_{u}^{(+)}(\gamma, \theta), \quad \tau>0 \\
Q^{(+)}(0, \theta, \gamma)= & \varphi_{2 r}\left(\lambda_{0}(\gamma), \theta\right) \sigma, \quad Q^{(+)}(+\infty, \theta, \gamma)=0 .
\end{aligned}
$$

Здесь $f_{u}^{(-)}(\gamma, \theta) \equiv f_{u}\left(\varphi_{1}\left(\lambda_{0}(\gamma), \theta\right), \lambda_{0}(\gamma), \theta, 0\right)$; остальные обозначения в $(4.11)$ те же, что в п. 4.1. Аналогично, $f_{u}^{(+)}(\gamma, \theta) \equiv f_{u}\left(\varphi_{2}\left(\lambda_{0}(\gamma), \theta\right), \lambda_{0}(\gamma), \theta, 0\right)$, а 
$f_{u}^{(+)}(\tau, \theta, \gamma)$ и $f_{r}^{(+)}(\tau, \theta, \gamma)$ обозначают производные функции $f$, вычисленные в точке $\left(\varphi_{2}\left(\lambda_{0}(\gamma), \theta\right)+\widetilde{Q}_{0}^{(+)}(\tau, \theta, \gamma), \lambda_{0}(\gamma), \theta, 0\right)$.

Как и при получении уравнения (4.6), можно показать, что

$$
\frac{\partial Q^{(-)}}{\partial \tau}(0, \theta, \gamma)-\frac{\partial Q^{(+)}}{\partial \tau}(0, \theta, \gamma)=-I(\gamma, \theta) \sigma+\Phi(\gamma, \theta)
$$

где $\Phi(\gamma, \theta)$ - известная функция. Возьмем столь большое $\sigma>0$, чтобы выполнялось неравенство

$$
\frac{\partial Q^{(-)}}{\partial \tau}(0, \theta, \gamma)-\frac{\partial Q^{(+)}}{\partial \tau}(0, \theta, \gamma)>0, \quad \gamma \in\left[-\gamma_{0}, \gamma_{0}\right]
$$

что возможно в силу неравенства (4.7). Теперь функции $Q^{( \pm)}\left(\tau_{\beta}, \theta, \gamma\right)$ полностью определены.

Умножим функции $\widetilde{Q}_{i}^{( \pm)}$, входящие в $U\left(x, \tau_{\beta}, \gamma, \varepsilon\right)$, а также $Q^{( \pm)}, P_{4}^{(-)}$, входящие в $(4.8)$ и (4.9), на соответствуюшие срезающие функции и определим функцию $v_{\beta}(r, \theta, \varepsilon)$, исходя из условия $\tilde{\beta}(x, \gamma, \varepsilon) \in C\left(\bar{D} \times\left[0, \gamma_{0}\right]\right)$, аналогично тому, как мы определили функцию $v_{n \beta}$ при доказательстве теоремы 3.2. Тогда $v_{\beta}=O\left(\varepsilon^{4}\right)$. Итак, функция $\tilde{\beta}(x, \gamma, \varepsilon)$ полностью определена.

Из (4.5) и (4.12) следует, что при достаточно малых $\varepsilon$

$$
\left.\frac{\partial \tilde{\beta}^{(-)}}{\partial r}\right|_{\Lambda_{\gamma, \beta}}-\left.\frac{\partial \tilde{\beta}^{(+)}}{\partial r}\right|_{\Lambda_{\gamma, \beta}} \geqslant 0, \quad \gamma \in\left[0, \gamma_{0}\right] .
$$

Обозначим через $\partial / \partial n_{\beta}$ производную по направлению внутренней нормали к кривой $\Lambda_{\gamma, \beta}$. Нетрудно показать, что

$$
\left[\left.\frac{\partial \tilde{\beta}^{(-)}}{\partial n_{\beta}}\right|_{\Lambda_{\gamma, \beta}}-\left.\frac{\partial \tilde{\beta}^{(+)}}{\partial n_{\beta}}\right|_{\Lambda_{\gamma, \beta}}\right]=(1+O(\varepsilon))\left[\left.\frac{\partial \tilde{\beta}^{(-)}}{\partial r}\right|_{\Lambda_{\gamma, \beta}}-\left.\frac{\partial \tilde{\beta}^{(+)}}{\partial r}\right|_{\Lambda_{\gamma, \beta}}\right] .
$$

Поэтому при достаточно малых $\varepsilon$

$$
\left.\frac{\partial \tilde{\beta}^{(-)}}{\partial n_{\beta}}\right|_{\Lambda_{\gamma, \beta}} \geqslant\left.\frac{\partial \tilde{\beta}^{(+)}}{\partial n_{\beta}}\right|_{\Lambda_{\gamma, \beta}}, \quad \gamma \in\left[0, \gamma_{0}\right] .
$$

Кроме того, функция $\tilde{\beta}(x, \gamma, \varepsilon)$ построена таким образом, что при достаточно малых $\varepsilon$ выполняются неравенства

$$
\begin{gathered}
\varepsilon^{2} \Delta \tilde{\beta}-f(\tilde{\beta}, x, \varepsilon)=-\varepsilon^{3} f_{u}\left(\varphi_{1}(x), x, 0\right)+O\left(\varepsilon^{4}\right)<-m_{\varepsilon}<0, \\
x \in \bar{\Omega}_{\gamma, \beta}^{(-)}, \quad \gamma \in\left[0, \gamma_{0}\right], \\
\varepsilon^{2} \Delta \tilde{\beta}-f(\tilde{\beta}, x, \varepsilon)=-\varepsilon^{3} f_{u}\left(\varphi_{2}(x), x, 0\right)+O\left(\varepsilon^{4}\right)<-m_{\varepsilon}<0, \\
x \in \bar{\Omega}_{\gamma, \beta}^{(+)}, \quad \gamma \in\left[0, \gamma_{0}\right],
\end{gathered}
$$

где

$$
m_{\varepsilon}=\frac{\varepsilon^{3}}{2} \min \left\{\min _{x \in \bar{D}} f_{u}\left(\varphi_{1}(x), x, 0\right), \min _{x \in \bar{D}} f_{u}\left(\varphi_{2}(x), x, 0\right)\right\}>0
$$


Наконец,

$$
\left.\frac{\partial \tilde{\beta}}{\partial n}\right|_{\partial D}=0, \quad \gamma \in\left[0, \gamma_{0}\right]
$$

Аналогично функции $\tilde{\beta}(x, \gamma, \varepsilon)$ определим функцию $\tilde{\alpha}(x, \gamma, \varepsilon)$ :

$$
\begin{aligned}
\tilde{\alpha}(x, \gamma, \varepsilon)= & \tilde{\alpha}^{(-)}(x, \gamma, \varepsilon)=U\left(x, \tau_{\alpha}, \gamma, \varepsilon\right)-\varepsilon^{3}\left(1+Q^{(-)}\left(\tau_{\alpha}, \theta, \gamma\right)\right) \\
& +P_{4}^{(-)}(\xi, \ell, \varepsilon), \quad x \in \bar{\Omega}_{\gamma, \alpha}^{(-)} \\
\tilde{\alpha}(x, \gamma, \varepsilon)= & \tilde{\alpha}^{(+)}(x, \gamma, \varepsilon)=U\left(x, \tau_{\alpha}, \gamma, \varepsilon\right)-\varepsilon^{3}\left(1+Q^{(+)}\left(\tau_{\alpha}, \theta, \gamma\right)\right) \\
& +v_{\alpha}(r, \theta, \varepsilon), \quad x \in \bar{\Omega}_{\gamma, \alpha}^{(+)}
\end{aligned}
$$

где $\tau_{\alpha}=\left(r-r_{\alpha}\right) / \varepsilon, r_{\alpha}=r_{\gamma}+\varepsilon^{3} \sigma, \sigma>0, \Omega_{\gamma, \alpha}^{(-)}$- внешняя, а $\Omega_{\gamma, \alpha}^{(+)}-$внутренняя подобласти области $D$ по отношению к кривой $\Lambda_{\gamma, \alpha}$, имеюшей в локальных координатах $(r, \theta)$ уравнение $r=r_{\alpha}, \gamma \in\left[-\gamma_{0}, 0\right]$.

Умножим функции $\widetilde{Q}_{i}^{( \pm)}, Q^{( \pm)}$и $P_{4}^{(-)}$на соответствуюшие срезаюшие функции и определим функцию $v_{\alpha}$ аналогично функции $v_{\beta}$.

Нетрудно показать, что функция $\tilde{\alpha}(x, \gamma, \varepsilon)$ при достаточно малых $\varepsilon$ удовлетворяет следуюшим соотношениям:

$$
\begin{aligned}
\varepsilon^{2} \Delta \tilde{\alpha}-f(\tilde{\alpha}, x, \varepsilon)= & \varepsilon^{3} f_{u}\left(\varphi_{1}(x), x, 0\right)+O\left(\varepsilon^{4}\right)>m_{\varepsilon}>0, \quad x \in \bar{\Omega}_{\gamma, \alpha}^{(-)}, \quad \gamma \in\left[-\gamma_{0}, 0\right], \\
\varepsilon^{2} \Delta \tilde{\alpha}-f(\tilde{\alpha}, x, \varepsilon)= & \varepsilon^{3} f_{u}\left(\varphi_{2}(x), x, 0\right)+O\left(\varepsilon^{4}\right)>m_{\varepsilon}>0, \quad x \in \bar{\Omega}_{\gamma, \alpha}^{(+)}, \quad \gamma \in\left[-\gamma_{0}, 0\right], \\
& \left.\frac{\partial \tilde{\alpha}^{(-)}}{\partial n_{\alpha}}\right|_{\Lambda_{\gamma, \alpha}} \leqslant\left.\frac{\partial \tilde{\alpha}^{(+)}}{\partial n_{\alpha}}\right|_{\Lambda_{\gamma, \alpha}}, \quad \gamma \in\left[-\gamma_{0}, 0\right], \\
\left.\frac{\partial \tilde{\alpha}}{\partial n}\right|_{\partial D}=0, \quad \gamma \in\left[-\gamma_{0}, 0\right], &
\end{aligned}
$$

где $m_{\varepsilon}$ определено формулой (4.17), $\partial / \partial n_{\alpha}$ - производная по направлению внутренней нормали к кривой $\Lambda_{\gamma, \alpha}$.

Для удобства записи введем обозначения:

$$
\beta_{\varepsilon}(x, \gamma) \equiv \tilde{\beta}(x, \gamma, \varepsilon), \quad \alpha_{\varepsilon}(x, \gamma) \equiv \tilde{\alpha}(x,-\gamma, \varepsilon), \quad \gamma \in\left[0, \gamma_{0}\right] .
$$

Рассмотрим свойства функций $\beta_{\varepsilon}$ и $\alpha_{\varepsilon}$.

Докажем, что для любого фиксированного $C>0$ при достаточно малых $\varepsilon$

$$
\beta_{\varepsilon}\left(x, \gamma_{0}\right)>u^{(1)}(x, \varepsilon)+C \varepsilon^{4}, \quad x \in \bar{D},
$$

где $u^{(1)}(x, \varepsilon)$ - решение задачи $(1.1),(1.2)$ из леммы 3.5 .

Введем область $H_{1}=\left\{M(r, \theta) \in D:-d \leqslant r \leqslant r_{\beta}^{0}-h\right\}$, где $r_{\beta}^{0}=r_{\gamma_{0}}-\varepsilon^{3} \sigma$, а $h>0$ - сколь угодно малое, но фиксированное при $\varepsilon \rightarrow 0$ число, и рассмотрим область $H_{1} \cup\left(\overline{D_{0}^{(-)} \backslash D^{d}}\right)$. В этой области в силу экспоненциальной оценки функции $\widetilde{Q}_{i}^{(-)}$и $Q^{(-)}$при достаточно малых $\varepsilon$ будут меньше любой степени $\varepsilon$, и поэтому, используя $(4.8)$ и (3.79), получаем

$$
\beta_{\varepsilon}\left(x, \gamma_{0}\right)-u^{(1)}(x, \varepsilon)=\varepsilon^{3}+O\left(\varepsilon^{4}\right), \quad x \in H_{1} \cup\left(\overline{D_{0}^{(-)} \backslash D^{d}}\right),
$$


откуда следует, что для любого фиксированного числа $C>0$ при достаточно малых $\varepsilon$ неравенство (4.20) выполняется для $x \in H_{1} \cup\left(\overline{D_{0}^{(-)} \backslash D^{d}}\right)$.

Далее рассмотрим область $H_{2}=\left\{M(r, \theta) \in D: \tilde{r} \leqslant r \leqslant r_{\beta}^{0}\right\}$, где $\tilde{r}=r_{\beta}^{0}-$ $k \varepsilon|\ln \varepsilon|, \quad k>0$ - число, которое выберем ниже. Так как $\widetilde{Q}_{0}^{(-)}\left(\tau_{\beta}, \theta, \gamma_{0}\right)$ имеет экспоненциальную оценку снизу:

$$
\widetilde{Q}_{0}^{(-)}\left(\tau_{\beta}, \theta, \gamma_{0}\right) \geqslant C_{1} \exp \left(m_{1} \tau_{\beta}\right),
$$

то в области $H_{2}$, т.е. при $-k|\ln \varepsilon| \leqslant \tau_{\beta} \leqslant 0$ при достаточно малых $\varepsilon$ имеем

$$
\widetilde{Q}_{0}^{(-)}\left(\tau_{\beta}, \theta, \gamma_{0}\right) \geqslant C_{1} \varepsilon^{k m_{1}} .
$$

Возьмем $k>0$ столь мальм, что $p=k m_{1}<1$. Тогда получим

$$
\beta_{\varepsilon}\left(x, \gamma_{0}\right)-u^{(1)}(x, \varepsilon) \geqslant C_{1} \varepsilon^{p}+O(\varepsilon), \quad x \in H_{2},
$$

откуда следует (4.20) для $x \in H_{2}$.

В области $H_{3}=\left\{M(r, \theta) \in D: r_{\beta}^{0}-h \leqslant r \leqslant \tilde{r}\right\}$ имеем $\beta_{\varepsilon}\left(x, \gamma_{0}\right)=\varphi_{1}(x)+O\left(\varepsilon^{s}\right)$, где $s \in(0,1), u^{(1)}(x, \varepsilon)=\varphi_{1}(x)+O(\varepsilon)$. Поэтому, вычитая из (4.15) уравнение $\varepsilon^{2} \Delta u^{(1)}-f\left(u^{(1)}, x, \varepsilon\right)=0$, нетрудно получить для функции

$$
v=\beta_{\varepsilon}\left(x, \gamma_{0}\right)-u^{(1)}(x, \varepsilon)-C \varepsilon^{4}
$$

с произвольной постоянной $C>0$ следующее неравенство:

$$
\varepsilon^{2} \Delta v-\left[f_{u}\left(\varphi_{1}(x), x, 0\right)+O\left(\varepsilon^{s}\right)\right] v<-\varepsilon^{3} f_{u}\left(\varphi_{1}(x), x, 0\right)+O\left(\varepsilon^{4}\right), \quad x \in H_{3} .
$$

При достаточно малых $\varepsilon$ из этого неравенства, неравенства $f_{u}\left(\varphi_{1}(x), x, 0\right)>0$ (см. (A1)) и граничного неравенства

$$
\left.v\right|_{\partial H_{3}}>0
$$

вытекающего из того факта, что (4.20) выполняется в $H_{1}$ и $H_{2}$, следует, что $v>0$ при $x \in H_{3}$, т.е. (4.20) выполняется в области $H_{3}$.

Таким образом, (4.20) доказано в области $\bar{\Omega}_{\gamma_{0}, \beta}^{(-)}$. В области $\bar{\Omega}_{\gamma_{0}, \beta}^{(+)}$для любой фиксированной постоянной $C>0$ при достаточно малых $\varepsilon$ неравенство (4.20) также имеет место, поскольку в указанной области $\beta_{\varepsilon}\left(x, \gamma_{0}\right)=\varphi_{2}(x)+\widetilde{Q}_{0}^{(+)}\left(\tau_{\beta}, \theta, \gamma_{0}\right)+$ $O(\varepsilon), u^{(1)}(x, \varepsilon)=\varphi_{1}(x)+O(\varepsilon)$ и $\varphi_{2}(x)+\widetilde{Q}_{0}^{(+)}\left(\tau_{\beta}, \theta, \gamma_{0}\right) \geqslant 0, \mathrm{a} \varphi_{1}(x)<0$.

Итак, (4.20) доказано.

Аналогично (4.21) в области $\overline{D_{0}^{(+)} \cup D^{\gamma_{0} / 2}}$, где $D^{\gamma_{0} / 2}=\left\{M(r, \theta) \in D:-\gamma_{0} / 2<\right.$ $r \leqslant 0\}$, получаем равенство

$$
\beta_{\varepsilon}\left(x, \gamma_{0}\right)-u^{(2)}(x, \varepsilon)=\varepsilon^{3}+O\left(\varepsilon^{4}\right),
$$

где $u^{(2)}(x, \varepsilon)$ - решение задачи (1.1), (1.2) из леммы 3.5.

Отсюда следует, что для любого $C>0$, не зависящего от $\varepsilon$, при достаточно малых $\varepsilon$ выполняется неравенство

$$
\beta_{\varepsilon}\left(x, \gamma_{0}\right)>u^{(2)}(x, \varepsilon)+C \varepsilon^{4}, \quad x \in \overline{D_{0}^{(+)} \cup D^{\gamma_{0} / 2}} .
$$


Воспользуемся теперь теоремой 3.2 , согласно которой (для $n=3$ и $d=\gamma_{0} / 2$ ) при достаточно малых $\varepsilon$ существуют числа $T_{5}=T_{5}\left(3, \gamma_{0} / 2, \varepsilon\right)$ и $\bar{C}_{3}>0$ такие, что

$$
\begin{array}{ll}
u\left(x, T_{5}, \varepsilon\right)<u^{(1)}(x, \varepsilon)+\bar{C}_{3} \varepsilon^{4}, & x \in \overline{D_{0}^{(-)} \backslash D^{\gamma_{0} / 2}}, \\
u\left(x, T_{5}, \varepsilon\right)<u^{(2)}(x, \varepsilon)+\bar{C}_{3} \varepsilon^{4}, & x \in \overline{D_{0}^{(+)} \cup D^{\gamma_{0} / 2}} .
\end{array}
$$

Поскольку число $C$ в $(4.20)$ и $(4.22)$ можно взять равньм числу $\bar{C}_{3}$ из неравенств $(4.23)$, то из $(4.20),(4.22)$ и (4.23), очевидно, следует, что при достаточно малых $\varepsilon$

$$
u\left(x, T_{5}, \varepsilon\right)<\beta_{\varepsilon}\left(x, \gamma_{0}\right), \quad x \in \bar{D} .
$$

Аналогично можно доказать неравенство

$$
u\left(x, T_{5}, \varepsilon\right)>\alpha_{\varepsilon}\left(x, \gamma_{0}\right), \quad x \in \bar{D} .
$$

ЛЕмма 4.1. Пусть выполнены условия (A1)-(А5). Тогда при достаточно малых в имеют место следующие утверждения:

1) существует $T^{\prime}=T^{\prime}(\varepsilon)>0$ такое, что решение $u(x, t, \varepsilon)$ задачи (1.1)(1.3) удовлетворяет неравенствам

$$
\alpha_{\varepsilon}(x, 0)<u(x, t, \varepsilon)<\beta_{\varepsilon}(x, 0), \quad(x, t) \in \bar{D} \times\left[T^{\prime},+\infty\right) ;
$$

2) существует стационарное решение $u_{s}(x, \varepsilon) \in C^{2}(\bar{D})$ задачи (1.1), (1.2), представляющее собой КСТС, т.е. удовлетворяющее предельному равенству (1.5), и такое, что

$$
\alpha_{\varepsilon}(x, 0)<u_{s}(x, \varepsilon)<\beta_{\varepsilon}(x, 0), \quad x \in \bar{D} .
$$

ДОКАЗАТЕЛЬСТВо. 1. Положим

$$
T_{\beta}=\frac{2 \gamma_{0}}{m_{\varepsilon}} \sup _{\bar{D} \times\left[0, \gamma_{0}\right]}\left|\frac{\partial \beta_{\varepsilon}}{\partial \gamma}(x, \gamma)\right|,
$$

где число $m_{\varepsilon}$ определено формулой (4.17).

Аналогично, заменив $\frac{\partial \beta_{\varepsilon}}{\partial \gamma}$ на $\frac{\partial \alpha_{\varepsilon}}{\partial \gamma}$, введем $T_{\alpha}$ и положим

$$
T=\max \left\{T_{\beta}, T_{\alpha}\right\}, \quad T^{\prime}=T+T_{5} ;
$$

здесь $T_{5}$ - то же число, что и в неравенствах (4.24), (4.25).

Введем функции

$$
\begin{gathered}
\hat{v}(x, t)=\beta_{\varepsilon}(x, \gamma(t)), \quad \bar{v}(x, t)=\alpha_{\varepsilon}(x, \gamma(t)), \quad(x, t) \in \bar{D} \times\left[T_{5}, T^{\prime}\right], \\
\gamma(t)=\frac{T^{\prime}-t}{T} \gamma_{0}, \quad t \in\left[T_{5}, T^{\prime}\right] .
\end{gathered}
$$

Докажем, что выполняются неравенства

$$
\begin{aligned}
\bar{v}(x, t) & <u(x, t)<\hat{v}(x, t), & & (x, t) \in \bar{D} \times\left[T_{5}, T^{\prime}\right], \\
\bar{v}\left(x, T^{\prime}\right) & <u(x, t)<\hat{v}\left(x, T^{\prime}\right), & & (x, t) \in \bar{D} \times\left[T^{\prime},+\infty\right) .
\end{aligned}
$$


Из неравенств (4.32) в силу (4.30) непосредственно следуют неравенства (4.26), т.е. утверждение 1$)$.

Докажем (4.31). Неравенства (4.32) можно доказать аналогичным образом, опираясь на неравенства (4.31), взятые при $t=T^{\prime}$.

В силу (4.24), (4.25), (4.30) выполнены неравенства

$$
\bar{v}\left(x, T_{5}\right)<u\left(x, T_{5}, \varepsilon\right)<\hat{v}\left(x, T_{5}\right), \quad x \in \bar{D} .
$$

Поэтому если (4.31) не выполнено, то существует $\widetilde{T} \in\left(T_{5}, T^{\prime}\right]$ такое, что

$$
\begin{gathered}
\bar{v}(x, t)<u(x, t, \varepsilon)<\hat{v}(x, t), \quad(x, t) \in \bar{D} \times\left[T_{5}, \widetilde{T}\right), \\
\exists M \in \bar{D}: \quad u(M, \widetilde{T}, \varepsilon)=\hat{v}(M, \widetilde{T}), \quad \text { либо } \quad u(M, \widetilde{T}, \varepsilon)=\bar{v}(M, \widetilde{T}) .
\end{gathered}
$$

Для определенности будем считать, что выполнено (4.34).

Введем функцию

$$
w(x, t)=(\hat{v}(x, t)-u(x, t, \varepsilon)) \exp \left(-A\left(t-T_{5}\right)\right),
$$

где $A$ - положительное число, которое будет выбрано ниже.

Простые вычисления с учетом (4.14)-(4.16), (4.18), (4.13), (4.28), (4.30) приводят к следуюшим соотношениям для функции $w$ :

$$
\begin{aligned}
\varepsilon^{2} \Delta w+B(x, t) w-\frac{\partial w}{\partial t} & \leqslant-\frac{m_{\varepsilon}}{2} \exp \left(-A\left(t-T_{5}\right)\right), \quad(x, t) \in \bar{D} \times\left(T_{5}, \widetilde{T}\right] \\
\left.\left(\frac{\partial w}{\partial r}\right)^{(-)}\right|_{\Lambda_{\gamma(t), \beta}} & \geqslant\left.\left(\frac{\partial w}{\partial r}\right)^{(+)}\right|_{\Lambda_{\gamma(t), \beta}}, \quad t \in\left(T_{5}, \widetilde{T}\right] \\
\left.\left(\frac{\partial w}{\partial n_{\beta}}\right)^{(-)}\right|_{\Lambda_{\gamma(t), \beta}} & \geqslant\left.\left(\frac{\partial w}{\partial n_{\beta}}\right)^{(+)}\right|_{\Lambda_{\gamma(t), \beta}, \quad t \in\left(T_{5}, \widetilde{T}\right]} \\
\left.\frac{\partial w}{\partial n}\right|_{\partial D} & =0, \quad t \in\left(T_{5}, \widetilde{T}\right]
\end{aligned}
$$

где

$$
B(x, t) \equiv-A-\int_{0}^{1} f_{u}(u+(\hat{v}-u) s, x, \varepsilon) d s,
$$

обозначения $(\cdot)^{(+)}$и $(\cdot)^{(-)}$использованы для предельных значений на $\Lambda_{\gamma(t), \beta}$ из областей $\Omega_{\gamma(t), \beta}^{(+)}$и $\Omega_{\gamma(t), \beta}^{(-)}$соответственно, а неравенство (4.35) на поверхности $\Lambda_{\gamma(t), \beta} \times\left(T_{5}, \widetilde{T}\right]$ выполняется для предельных значений левой части неравенства.

Выберем $A>0$ столь большим, что

$$
B(x, t)<0, \quad(x, t) \in \bar{D} \times\left[T_{5}, \widetilde{T}\right] .
$$

Из (4.33), (4.34) следует, что

$$
\begin{gathered}
w(x, t)>0, \quad(x, t) \in \bar{D} \times\left[T_{5}, \widetilde{T}\right), \\
\exists M \in \bar{D}: \quad w(M, \widetilde{T})=0 .
\end{gathered}
$$


Пусть $M \in \Lambda_{\gamma(\widetilde{T}), \beta}$. Возьмем число $h>0$ столь малым, что круг $E_{h}$ радиуса $h$ с центром в точке $M$ целиком принадлежит области $D^{d}$ (см. формулировку теорем $3.1,3.2)$. Введем обозначения $w_{M}(r, t) \equiv w\left(x\left(r, \theta_{M}\right), t\right)$, где $\theta_{M}-\theta$-координата точки $M$, и

$$
W^{(-)}(t) \equiv w_{M}\left(r_{\beta}(t)-0, t\right), \quad W^{(+)}(t) \equiv w_{M}\left(r_{\beta}(t)+0, t\right),
$$

где $r_{\beta}(t)=\left.r_{\gamma(t)}\right|_{\theta=\theta_{M}}-\varepsilon^{3} \sigma$. В силу непрерывности функции $w_{M}(r, t)$

$$
W^{(-)}(t)=W^{(+)}(t), \quad t \in\left[T_{5}, \widetilde{T}\right],
$$

а следствием (4.39), (4.40) является неравенство

$$
W_{t}^{( \pm)}(\widetilde{T}) \leqslant 0 .
$$

Докажем, что

$$
\begin{aligned}
w_{t}^{(-)} & \equiv \frac{\partial w_{M}}{\partial t}\left(r_{\beta}(\widetilde{T})-0, \widetilde{T}\right) \leqslant 0, \\
w_{t}^{(+)} & \equiv \frac{\partial w_{M}}{\partial t}\left(r_{\beta}(\widetilde{T})+0, \widetilde{T}\right) \leqslant 0 .
\end{aligned}
$$

При этом будем пользоваться тем, что в силу (4.36)

$$
\frac{\partial w_{M}}{\partial r}\left(r_{\beta}(\widetilde{T})-0, \widetilde{T}\right) \equiv w_{r}^{(-)} \geqslant w_{r}^{(+)} \equiv \frac{\partial w_{M}}{\partial r}\left(r_{\beta}(\widetilde{T})+0, \widetilde{T}\right),
$$

а также тем, что в силу (4.2), (4.10), (4.30) при достаточно малых $\varepsilon$

$$
\frac{d r_{\beta}}{d t}(t)=\frac{\partial r_{\beta}}{\partial \gamma}\left(\frac{\partial \gamma}{\partial t}\right)=(-1+O(\varepsilon))\left(-\frac{\gamma_{0}}{T}\right)>0, \quad t \in\left[T_{5}, \widetilde{T}\right] .
$$

В силу $(4.39),(4.40) w_{r}^{(+)} \geqslant 0$, а так как

$$
W_{t}^{( \pm)}(\widetilde{T})=w_{r}^{( \pm)} \frac{d r_{\beta}}{d t}(\widetilde{T})+w_{t}^{( \pm)}
$$

то отсюда и из соотношений (4.42), (4.45) следует, что

$$
w_{t}^{(+)} \leqslant 0
$$

Из (4.41) и (4.46) имеем

$$
w_{t}^{(-)}=w_{t}^{(+)}-\frac{d r_{\beta}}{d t}(\widetilde{T})\left(w_{r}^{(-)}-w_{r}^{(+)}\right)
$$

Отсюда в силу соотношений $(4.44),(4.45),(4.47)$ имеем

$$
w_{t}^{(-)} \leqslant 0
$$

и тем самым (4.43) доказано.

Выберем теперь $h>0$ столь мальм, что

$$
\varepsilon^{2} \Delta \bar{w}+\bar{B}(x) \bar{w}<-\frac{m_{\varepsilon}}{4} \exp \left(-A\left(\widetilde{T}-T_{5}\right)\right)<0, \quad x \in\left(E_{h} \backslash M\right),
$$


где $\bar{w}(x) \equiv w(x, \widetilde{T}), \bar{B}(x) \equiv B(x, \widetilde{T})<0$. Это можно сделать в силу (4.35), (4.43). Из принципа максимума для слабых решений эллиптических уравнений [11] следует, что соотношение (4.37), взятое при $t=\widetilde{T},(4.48)$ и неравенство $\bar{w}(x) \geqslant 0$, справедливое при $x \in E_{h}$ в силу (4.39), (4.40), противоречат нашему предположению $\bar{w}(M)=0$.

Если предположить, что точка $M$ в (4.34) не принадлежит кривой $\Lambda_{\gamma(\widetilde{T}), \beta}$, то противоречие можно получить аналогичным образом с помощью классического принципа максимума для решений эллиптических уравнений. Полученное противоречие доказывает справедливость (4.31).

Утверждение 1) доказано.

2. Утверждение 2) следует из того факта, что в силу (4.14)-(4.16), (4.18) и аналогичных неравенств для $\alpha_{\varepsilon}$ функции $\beta_{\varepsilon}(x, 0)$ и $\alpha_{\varepsilon}(x, 0)$ являются соответственно (негладкими) верхним и нижним решениями задачи (1.1), (1.2), причем, как показывает (4.26),

$$
\alpha_{\varepsilon}(x, 0)<\beta_{\varepsilon}(x, 0), \quad x \in \bar{D}
$$

(см. также [2], [6]). Лемма доказана.

ЗАмЕчАнИЕ. Очевидно, что для справедливости утверждения 2) леммы 4.1 достаточно лишш условий (A1), (A2), т.е. условий (А3)-(А5) не требуется.

\section{3. Предельный переход при $t \rightarrow+\infty$.}

ТЕОРема 4.1. Пусть выполнены условия (A1)-(А5). Тогда при достаточно мальх $\varepsilon$ для решения $u(x, t, \varepsilon)$ задачи (1.1)-(1.3) справедливо предельное равенство

$$
\lim _{t \rightarrow+\infty}\left\|u(x, t, \varepsilon)-u_{s}(x, \varepsilon)\right\|_{C(\bar{D})}=0,
$$

где $u_{s}(x, \varepsilon)$ - стационарное решение задачи (1.1), (1.2) из леммы 4.1.

ДокАЗАТЕЛЬСТво. Введем функции

$$
\begin{aligned}
& \hat{v}_{\varepsilon}(x, t) \equiv u_{s}(x, \varepsilon)+\Theta(x, \varepsilon) \exp (-\varepsilon \mu t), \\
& \bar{v}_{\varepsilon}(x, t) \equiv u_{s}(x, \varepsilon)-\Theta(x, \varepsilon) \exp (-\varepsilon \mu t),
\end{aligned}
$$

где $\Theta(x, \varepsilon) \equiv \beta_{\varepsilon}(x, 0)-\alpha_{\varepsilon}(x, 0)$, функции $\beta_{\varepsilon}$ и $\alpha_{\varepsilon}-$ те же, что в лемме $4.1, \mu>0-$ не зависящее от $\varepsilon$ число, которое будет уточнено ниже.

Из неравенств (4.26) и (4.27) следует, что

$$
\bar{v}_{\varepsilon}(x, 0)<u\left(x, T^{\prime}, \varepsilon\right)<\hat{v}_{\varepsilon}(x, 0), \quad x \in \bar{D} .
$$

Далее, используя подход, аналогичный предложенному при доказательстве теоремы 3.2 в отношении функции $\hat{v}$, нетрудно установить, что для любого достаточно малого $\mu$ при достаточно малых $\varepsilon$ функции $\bar{v}_{\varepsilon}\left(x, t-T^{\prime}\right)$ и $\hat{v}_{\varepsilon}\left(x, t-T^{\prime}\right)$ являются соответственно нижним и верхним барьерами для решения $u(x, t, \varepsilon)$ задачи $(1.1)-(1.3)$ при $t \geqslant T^{\prime}$, т.е.

$$
\bar{v}_{\varepsilon}\left(x, t-T^{\prime}\right)<u(x, t, \varepsilon)<\hat{v}_{\varepsilon}\left(x, t-T^{\prime}\right), \quad(x, t) \in \bar{D} \times\left[T^{\prime},+\infty\right)
$$

(см. также [6]).

Зафиксируем такое $\mu>0$, чтобы при достаточно малых $\varepsilon$ было выполнено (4.50). Из неравенств (4.50), очевидно, следует (4.49). Теорема доказана. 


\section{§ 5. Глобальная область влияния (основной результат)}

Пусть при $\varepsilon \in\left(0, \varepsilon^{\prime}\right]$, где $\varepsilon^{\prime}>0$ - некоторое число, задача $(1.1),(1.2)$ имеет стационарное решение $u_{\varepsilon}(x) \in C^{2}(\bar{D})$.

Введем множество $G\left(u_{\varepsilon}\right)$ следующим образом: $u_{0}(x, \varepsilon) \in G\left(u_{\varepsilon}\right)$, если $\exists \varepsilon^{\prime \prime} \in$ $\left(0, \varepsilon^{\prime}\right]$ такое, что при $\varepsilon \in\left(0, \varepsilon^{\prime \prime}\right]$ сушествует решение $u_{\varepsilon}(x, t) \in C^{1,0}(\bar{D} \times[0,+\infty)) \cap$ $C^{2,1}(\bar{D} \times(0,+\infty))$ задачи $(1.1)-(1.3)$ и $\left\|u_{\varepsilon}(x, t)-u_{\varepsilon}(x)\right\|_{C(\bar{D})} \rightarrow 0$ при $t \rightarrow+\infty$.

Заметим, что $u_{\varepsilon}(x) \in G\left(u_{\varepsilon}\right)$, а потому $G\left(u_{\varepsilon}\right)$ всегда непусто.

ОПРЕДЕЛЕНИЕ. Множество $G\left(u_{\varepsilon}\right)$ назовем глобальной областью влияния стачионарного решения $u_{\varepsilon}(x)$ задачи $(1.1),(1.2)$.

Теперь, используя результаты $\S 4$, можно сформулировать основной результат настоящей работы.

ТЕОРема 5.1. Пусть выполнены условия (A1) и (A2). Тогда при достаточно малых в существует стационарное решение $u_{s}(x, \varepsilon)$ из $C^{2}(\bar{D})$ задачи (1.1), (1.2), представляющее собой КСТС, т.е. удовлетворяющее предельному равенству (1.5). При выполнении условия (АЗ) в отношении глобальной области влияния $G\left(u_{s}\right)$ этого решения справедливы следующие утверэсдения:

1) функция $u_{0}(x, \varepsilon) \equiv u_{0}(x)$ принадлежит $G\left(u_{s}\right)$, если она удовлетворяет условию (A4) и условию (A5);

2) $u_{0}(x, \varepsilon) \in G\left(u_{s}\right)$, если существует $\varepsilon_{1}>0$ такое, что $u_{0}(x, \varepsilon) \in C_{B}^{2}(\bar{D})$ при $\varepsilon \in\left(0, \varepsilon_{1}\right]$ (см. (A4)) и существуют функиии $\bar{u}_{0}(x)$ и $\hat{u}_{0}(x)$, удовлетворяющие условиям (A4), (A5) и такие, что

$$
\bar{u}_{0}(x)<u_{0}(x, \varepsilon)<\hat{u}_{0}(x), \quad x \in \bar{D}, \quad \varepsilon \in\left(0, \varepsilon_{1}\right]
$$

Утверждение 2) является следствием утверждения 1) и результатов работ [8], [9].

СЛЕДСТВИЕ 5.1. Пусть выполнены условия (A1), (A2), (A4) и условия:

$(\mathrm{A} 2)^{\prime} \quad J(x)>0$ nрu $x \in D_{0}^{(-)} \cup \partial D, \quad J(x)<0$ nрu $x \in D_{0}^{(+)}$;

$(\mathrm{A} 5)^{\prime}$ существуют $x^{(-)} \in D_{0}^{(-)} u x^{(+)} \in D_{0}^{(+)}$mакие, что $u_{0}\left(x^{(-)}\right)<0$, $u_{0}\left(x^{(+)}\right)>0$.

Тогда $u_{0}(x) \in G\left(u_{s}\right)$.

ТЕОРемА 5.2. Пусть выполнены условия (A1), (A2), (A4) и одно из следующих условий:

(A6) $u_{0}(x)>0, \quad x \in \bar{D}_{0}^{(-)}\left(u_{0}(x)<0, x \in \bar{D}_{0}^{(+)}\right)$;

(A6)' cуществует $x_{\ell} \in D_{0}^{(-)}$такое, что $u_{0}\left(x_{\ell}\right)>0$ u $J\left(x_{\ell}\right)<0$ (существует $x_{r} \in D_{0}^{(+)}$такое, ито $u_{0}\left(x_{r}\right)<0$ u $\left.J\left(x_{r}\right)>0\right)$.

Тогда существуют $\eta>0, \quad T \geqslant 0 u \bar{x} \in \bar{D}$ такие, что при достаточно малых $\varepsilon$

$$
\left|u(\bar{x}, t, \varepsilon)-u_{s}(\bar{x}, \varepsilon)\right| \geqslant \eta, \quad t \in[T,+\infty),
$$

m.e. $u_{0}(x) \notin G\left(u_{s}\right)$.

Утверждение теоремы 5.2 непосредственно следует из леммы 3.4. 


\section{Список литературы}

1. Фай малым параметром // УМН. 1974. Т. 29. № 4. С. 103-131.

2. Нефедов H. H. Метод дифференциальных неравенств для некоторых классов нелинейных сингулярно возмущенных задач с внутренними слоями // Дифференц. уравнения. 1995. T. 31. № 7. C. 1132-1139.

3. Васильева А.Б., Бутузов В. Ф. Асимптотические методы в теории сингулярных возмущений. М.: Высш. шк., 1990.

4. Бутузов В. Ф., Васильева А. Б., Нефедов Н. Н. Контрастные структуры в сингулярно возмущенных задачах // Автоматика и телемеханика. 1997. Т. 11. № 7. С. 4-32.

5. Бутузов В. Ф., Неделько И. В. Устойчивость контрастных структур типа ступеньки в двумерном случае // Докл. РАН. 1999. Т. 366. № 3. С. 295-298.

6. Бутузов В.Ф., Неделько И. В. Асимптотическая устойчивость решений сингулярно возмущенных краевых задач с пограничными и внутренними слоями // Дифференц. уравнения. 2000. Т. 36. № 2. С. 198-208.

7. Бутузов В. Ф., Неделько И. В. О глобальной области влияния решений с внутренними слоями // Докл. РАН. 2000. Т. 373. № 2. С. 155-156.

8. Amann H. Periodic solutions of semilinear parabolic equations // Nonlinear Analysis: Collection of Papers in Honor of Erich Rothe. N. Y.: Academic Press, 1978. P. 1-29.

9. Amann $H$. Existence and stability of solutions for semilinear parabolic systems and applications to some diffusion reaction equations // Proc. Roy. Soc. of Edinburgh. 1978. V. 81. № 1. P. $35-47$.

10. Нефедов Н. Н. Метод дифференциальных неравенств для некоторых сингулярно возмущенных задач в частных производных // Дифференц. уравнения. 1995. Т. 31. №4. C. $719-722$.

11. Гилбарг Д., Трудингер Н. Эллиптические дифференциальные уравнения с частными производными второго порядка. М.: Наука, 1989.

Поступило в редакцию 20.III.2001 